\title{
ON LAGUERRE-HAHN ORTHOGONAL POLYNOMIALS ON THE REAL LINE
}

\author{
M.N. REBOCHO
}

\begin{abstract}
A survey is given on sequences of orthogonal polynomials related to Stieltjes functions satisfying a Riccati type differential equation with polynomial coefficients - the so-called Laguerre-Hahn class. The main goal is to describe analytical aspects, focusing on differential equations for those orthogonal polynomials, difference and differential equations for the recurrence coefficients, and distributional equations for the corresponding linear functionals.
\end{abstract}

KEYwords: Orthogonal polynomials; Stieltjes function; Laguerre-Hahn class; discrete Painlevé equations; matrix Sylvester equations.

Math. Subject Classification (2000): 33C45, 33C47, 42C05.

\section{Introduction}

1.1. Stieltjes functions and orthogonal polynomials. This paper surveys sequences of Laguerre-Hahn orthogonal polynomials, that is, sequences of orthogonal polynomials whose Stieltjes function, $S$, satisfies a Riccati differential equation

$$
A S^{\prime}=B S^{2}+C S+D,
$$

where $A, B, C, D$ are polynomials, $A \not \equiv 0$. Essentially, we will consider orthogonality on the real line.

A fairly general way to define the Stieltjes function is through the sequence of moments of a linear functional, say $u$, defined in the linear space of polynomials, where we shall assume the regularity condition on the moments $u_{n}:=\left\langle u, x^{n}\right\rangle, n \geq 0$,

$$
\operatorname{det}\left(\left(u_{i+j}\right)_{i, j=0}^{n}\right) \neq 0, \quad n \geq 0
$$

Received March 21, 2019.

This work is partially supported by the Centre for Mathematics of the University of Coimbra - UID/MAT/00324/2013, funded by the Portuguese Government through FCT/MCTES and cofunded by the European Regional Development Fund through the Partnership Agreement PT2020. Special thanks go to Alphonse P. Magnus, for useful comments and for pointing out some bibliographic references. 
and, without loss of generality, $u_{0}=1$. The Stieltjes function of $u$ is the formal series

$$
S(x)=\sum_{n=0}^{+\infty} \frac{u_{n}}{x^{n+1}} .
$$

If the moments satisfy the condition $\operatorname{det}\left(\left(u_{i+j}\right)_{i, j=0}^{n}\right)>0, n \geq 0$ (that is, $u$ is positive-definite), then $u$ has an integral representation in terms of a positive Borel measure, $\mu$, supported on an infinite point set of the real line, $I$, such that

$$
\left\langle u, x^{n}\right\rangle=\int_{I} x^{n} d \mu(x), \quad n \geq 0 .
$$

In this case, $S$ is the so-called Stieltjes transform of the measure [80],

$$
S(x)=\int_{I} \frac{d \mu(y)}{x-y}, \quad x \in \mathbb{C} \backslash I .
$$

The Stieltjes function plays a key role in the theory of orthogonal polynomials. In many problems, the function $S$ has more simple analytical and transformation properties than the measure or the related linear functional, thus, $S$ is often more convenient for analysis [90]. Indeed, in the work of Stieltjes (see $[79,80,83]$ ), often the Stieltjes transform of the measure is given instead of the measure itself. Note that the measure can be restored by means of an inversion formula (see [87, pp. 175] and [89]),

$$
\frac{1}{2} \mu(\{a\})+\frac{1}{2} \mu(\{b\})+\mu(] a, b[)=\lim _{\epsilon \rightarrow 0^{+}} \frac{1}{2 \pi i} \int_{a}^{b}(S(y-i \epsilon)-S(y+i \epsilon)) d y .
$$

The interplay between Stieltjes functions and orthogonal polynomials can be made through several ways. For instance, we may consider the orthogonality with respect to moment linear functionals defined on the algebra $\mathbb{P}$ of polynomials with complex coefficients (see [25, Th. 3.1]): condition (2) is a necessary and sufficient condition for the existence of a sequence of orthogonal polynomials with respect to the moment linear functional $u$ with moments $\left(u_{n}\right)_{n \geq 0}$, that is, for the existence of a sequence $\left\{P_{n}\right\}_{n \geq 0}$, with $\operatorname{deg}\left(P_{n}\right)=n, n \geq 0$, such that the orthogonality relation holds,

$$
\left\langle u, P_{n} P_{m}\right\rangle=h_{n} \delta_{n, m}, \quad n, m \geq 0,
$$

with $h_{n} \neq 0, n \geq 0$. Here, $\delta_{n, m}$ is the Kronecker's delta. In the positivedefinite case, with $u$ given through (4), the orthogonality condition (7) reads 


$$
\int_{I} P_{n}(x) P_{m}(x) d \mu(x)=h_{n} \delta_{n, m}, \quad h_{n}>0, \quad n, m \geq 0 .
$$

Throughout the paper we shall also use the terminology "the linear functional (or the measure) related to" a sequence of polynomials $\left\{P_{n}\right\}_{n \geq 0}$, or vice-versa, to express relations of type (7) or (8). In the account of the above setting, we shall also say that the Stieltjes function satisfying (1) is Laguerre-Hahn.

Throughout the paper we shall take each $P_{n}$ monic, that is, $P_{n}(x)=x^{n}+$ lower degree terms, and we will abbreviate the sequences of monic orthogonal polynomials by SMOP. Monic orthogonal polynomials satisfy a three-term recurrence relation $[82]$

$$
P_{n+1}(x)=\left(x-\beta_{n}\right) P_{n}(x)-\gamma_{n} P_{n-1}(x), \quad n=0,1,2, \ldots,
$$

with initial conditions $P_{-1}(x)=0, P_{0}(x)=1$, and $\gamma_{n} \neq 0, n \geq 1$. The converse result, usually referred to as Favard's Theorem (see [41, Th. 1.5] and [81, Th. 10.27]) states that, for the polynomial solution of (9) with $\gamma_{n} \neq 0$ (respectively, $\gamma_{n}>0, \beta_{n} \in \mathbb{R}$ ), and initial conditions $P_{-1}(x)=0, P_{0}(x)=1$, there exists a moment functional $u$ such that (7) holds (respectively, there exists a measure $\mu$ such that (8) holds).

The numbers $\beta_{n}, \gamma_{n}$, commonly called the recurrence coefficients, can be expressed as

$$
\beta_{n}=\frac{\left\langle u, x P_{n}^{2}\right\rangle}{h_{n}}, \quad \gamma_{n+1}=\frac{h_{n+1}}{h_{n}}, \quad n \geq 0 .
$$

In the pioneering work by Stieltjes (see $[2,79,80]$ ), the starting point in constructing the general theory of orthogonal polynomials is the representation of $S$ in terms of a continued fraction,

$$
S(x)=\frac{1}{x-\beta_{0}-\frac{\gamma_{1}}{x-\beta_{1}-\frac{\gamma_{2}}{\ddots}}} .
$$

The general theory of continued fraction shows that the approximants are rational functions whose numerators and denominators, here denoted by $P_{n-1}^{(1)} / P_{n}$, satisfy the recurrence relations, for all $n \geq 1$,

$$
\begin{gathered}
P_{n}^{(1)}(x)=\left(x-\beta_{n}\right) P_{n-1}^{(1)}(x)-\gamma_{n} P_{n-2}^{(1)}(x), \\
P_{n+1}(x)=\left(x-\beta_{n}\right) P_{n}(x)-\gamma_{n} P_{n-1}(x),
\end{gathered}
$$


with initial conditions $P_{-1}^{(1)}(x)=0, P_{0}^{(1)}(x)=1$ and $P_{-1}(x)=0, P_{0}(x)=1$. If $S$ is the Stieltjes transform of some measure $\mu$, then $\left\{P_{n}\right\}_{n \geq 0}$ is the sequence of orthogonal polynomials with respect to $\mu$ or, equivalently, to the moments (4) in the expansion (3).

The sequence $\left\{P_{n}^{(1)}\right\}_{n \geq 0}$ is nowadays commonly called the sequence of associated polynomials of the first kind, or simply the first-associated polynomials of $\left\{P_{n}\right\}_{n \geq 0}$ [86]. Note that $\left\{P_{n}^{(1)}\right\}_{n \geq 0}$ is defined by a three-term recurrence relation with recurrence coefficients given by the shift $n \rightarrow n+1$ on the recurrence coefficients of $\left\{P_{n}\right\}_{n \geq 0}$. According to Favard's Theorem, $\left\{P_{n}^{(1)}\right\}_{n \geq 0}$ is a sequence of orthogonal polynomials. The associated polynomials will play a fundamental role in the sequel: they appear in fundamental formulae on transformations of orthogonal polynomials (see [64, 66, 90]), and they are a basic tool for deriving differential equations for Laguerre-Hahn orthogonal polynomials (see [29, chapter IV], [34]). More details on these topics will be given in Section 1.3 as well as in Section 3.

1.2. The Laguerre-Hahn class - early references. The study of orthogonal polynomials related to Stieltjes functions satisfying Riccati differential equations can be traced back to the framework of Hermite-Padé approximation. We refer the interested reader to the work by A.P. Magnus [58], together with the references therein, where it is studied the so-called Riccati model for continued fractions (11). Some aspects of convergence of such a model are based on the behaviour of the related orthogonal polynomials as well as on differential equations that those polynomials satisfy. Indeed, it is of importance the technique (according to the author in [58], based on studies by Laguerre and Hahn $[46,50])$ that gives linear differential equations of fixed but arbitrary order, with polynomial coefficients of bounded but arbitrary degree, from the Riccati differential equation for the Stieltjes function. The terminology Laguerre-Hahn class is then used in A.P. Magnus' paper (see [58, pp. 222]), when conjecturing that sequences of orthogonal polynomials satisfying such a kind of differential equations are always associated to solutions of a Riccati differential equation with polynomial coefficients.

Shortly after [58] there are several works on Laguerre-Hahn orthogonal polynomials that we would like to emphasize: [29, 34, 61]. In these works it is detailed the formalism giving the common operations on linear functionals, together with the appropriate topological framework, to define the so-called Laguerre-Hahn linear functionals (or linear forms, in the terminology of the 
authors therein). It is given the following characterization [29, Th. 3.1]: the equivalence between the Riccati differential equation for the Stieltjes function, $A S^{\prime}=B S^{2}+C S+D$, and the distributional equation for the corresponding linear functional $u$,

$$
\mathcal{D}(A u)=\psi u+B\left(x^{-1} u^{2}\right), \quad \psi=A^{\prime}+C,
$$

with $D$ the polynomial defined in terms of $A, B, C$ by $[29$, p. 67] *

$$
D(x)=\left(u \theta_{0} A\right)^{\prime}(x)-\left(u \theta_{0}\left(A^{\prime}+C\right)\right)(x)-\left(u^{2} \theta_{0}^{2} B\right)(x) .
$$

Here, the left product of $u$ by a polynomial is defined as $\langle g u, p\rangle=\langle u, g p\rangle, g, p \in$ $\mathbb{P}$; the derivative $\mathcal{D} u$ is defined as $\langle\mathcal{D} u, p\rangle=-\left\langle u, p^{\prime}\right\rangle, p \in \mathbb{P}$; the functional $x^{-1} u$ and the product of two linear functionals are defined, respectively, as follows: $\left\langle x^{-1} u, p\right\rangle=\left\langle u, \theta_{0} p\right\rangle$, with $\left(\theta_{c} p\right)(x)=\frac{p(x)-p(c)}{x-c}, c \in \mathbb{R}$, and $\langle u v, p\rangle=$ $\langle u, v p\rangle, p \in \mathbb{P}$, with the right product given by $v p(x)=\left\langle v, \frac{x p(x)-t p(t)}{x-t}\right\rangle$.

Note that $B \equiv 0$ in the previous equations gives us the semi-classical case $[48,60,62,63]$. In the semi-classical case, when $u$ is represented in terms of a weight function, $w$, that is,

$$
\left\langle u, x^{n}\right\rangle=\int_{I} x^{n} w(x) d x, n \geq 0,
$$

the distributional equation $\mathcal{D}(A u)=\psi u$ is equivalent to the equation for the weight $A w^{\prime}=C w$ - the so-called Pearson equation, under the boundary conditions

$$
\left.x^{n} A(x) w(x)\right|_{a, b}=0, \quad n \geq 0 .
$$

The numbers $a, b$ (eventually $a$ or $b$ infinite) are related to the roots of $A$. In such a case, $w$ is the weight function on the support $I=[a, b]$.

Amongst the semi-classical polynomials, there is the well-known family obtained when $\operatorname{deg}(A) \leq 2, \operatorname{deg}(C)=1$ - the so-called classical orthogonal polynomials - the Hermite, Laguerre, Jacobi, and Bessel polynomials. Let us us emphasize that the Hermite, Laguerre and Jacobi polynomials correspond to positive-definite cases, whilst the Bessel polynomials correspond to a quasi-definite case. For further purposes, mainly for an easier understanding of some of the results to be described, it is given a table, in the

\footnotetext{
*In these formulae, as well as in the rest of the paper, we are using our notation. It differs from $[29,34,61]$ and from other Maroni's works, from a minus sign on the polynomials $B$ and $D$, as the definition of Stieltjes function in these references is $S(x)=-\sum_{n \geq 0} u_{n} x^{-n-1}$.
} 
Appendix, with the information on the polynomials $A, C, D$ in the linear differential equation for the Stieltjes function, together with the weight and the recurrence coefficients for classical orthogonal polynomials.

The study of linear functionals satisfying a distributional equation of type (13) has been revisited many times in the literature. The main topics concern classification, integral representations, and functional perturbations (see [3, $4,12,13,68,69]$ ). More details about these topics will be given in the forthcoming sections.

\subsection{Building (some) sequences of Laguerre-Hahn orthogonal po-} lynomials, and related topics. Many families of Laguerre-Hahn orthogonal polynomials usually appear when dealing with transformations of semiclassical orthogonal polynomials. Essentially, two types of transformations are considered: (i) perturbations of the recurrence coefficients of orthogonal polynomials; (ii) rational spectral transformations of Stieltjes functions.

The literature on the above type of transformations, within the general theory of orthogonal polynomials, is vast, and clearly extends the topics covered in the present paper. We refer the interested reader to [65, 66, 76, 90] and their list of references.

Returning to the examples of Laguerre-Hahn orthogonal polynomials, let us begin with the probably most basic one: the $k$ th associated polynomials of semi-classical orthogonal polynomials. Recall that given a sequence of orthogonal polynomials, say $\left\{P_{n}\right\}_{n \geq 0}$, satisfying the recurrence relation (9), one defines, for $k \in \mathbb{N}$, the $k$ th associated polynomials $P_{n}^{(k)}, n=0,1, \ldots$, by the recurrence relation

$$
P_{n+1}^{(k)}(x)=\left(x-\beta_{n+k}\right) P_{n}^{(k)}(x)-\gamma_{n+k} P_{n-1}^{(k)}(x), \quad n=0,1,2, \ldots,
$$

with initial conditions $P_{-1}^{(k)}(x)=0, P_{0}^{(k)}(x)=1$. The Stieltjes function of the $k$ th associated polynomials is

$$
S^{(k)}(x)=\frac{1}{x-\beta_{k}-\frac{\gamma_{k+1}}{x-\beta_{k+1}-\frac{\gamma_{k+2}}{\ddots}}} .
$$


Thus, in the account of $(11), S^{(k)}$ is a rational spectral transformation of the Stieltjes function $S$ of $\left\{P_{n}\right\}_{n \geq 0}$, given by [90, Sec. 4]

$$
S^{(k)}(x)=\frac{P_{k}(x) S(x)-P_{k-1}^{(1)}(x)}{\gamma_{k} P_{k-1}(x) S(x)-\gamma_{k} P_{k-2}^{(1)}(x)} .
$$

Taking into account the above formulae, if $\left\{P_{n}\right\}_{n \geq 0}$ is semi-classical with $S$ satisfying a linear differential equation with polynomial coefficients, then, by virtue of (15), the Riccati equation for $S^{(k)}$ follows. Thus, if no simplifications occur, $\left\{P_{n}^{(k)}\right\}_{n \geq 0}$ is Laguerre-Hahn. Following this line, the most basic examples of Laguerre-Hahn orthogonal polynomials are the associated of Hermite, Laguerre, Jacobi and Bessel polynomials ${ }^{\dagger}$.

We can also consider the backward shift of the recurrence coefficients of $\left\{P_{n}\right\}_{n \geq 0}$, that is, one defines the new sequence of orthogonal polynomials, the so-called anti-associated polynomials $P_{n}^{(-k)}, n=0,1, \ldots$, , with recurrence coefficients [76]

$$
\beta_{n}^{(-k)}=\beta_{n-k}, \quad n=k, k+1, \ldots, \quad \gamma_{n}^{(-k)}=\gamma_{n-k}, \quad n=k+1, k+2, \ldots,
$$

where the parameters $\beta_{n}^{(-k)}, n=0, \ldots, k-1$, and $\gamma_{n}^{(-k)}, n=1, \ldots, k$, are defined as arbitrary numbers. In the same way as above, the Stieltjes function of $\left\{P_{n}^{(-k)}\right\}_{n \geq 0}$, here denoted by $S^{(-k)}$, is a spectral transformation of $S$ [90, Sec. 4],

$$
S^{(-k)}(x)=\frac{\gamma_{k}^{(-k)} P_{k-2}^{(-k)}(x) S(x)-P_{k-1}^{(-k+1)}(x)}{\gamma_{k}^{(-k)} P_{k-1}^{(-k)}(x) S(x)-P_{k}^{(-k+1)}(x)} .
$$

Transformations (15) and (16) are special cases of general rational spectral transformations

$$
\tilde{S}(x)=\frac{a(x) S(x)+b(x)}{c(x) S(x)+d(x)},
$$

where $a, b, c, d$ are polynomials [90]. Other relevant special cases of (17) are related to the following perturbations of orthogonal polynomials (see [28]): the generalized co-recursive polynomials, where it is modified, at level $k_{1}$, the coefficient $\beta_{k_{1}}$,

$$
\beta_{k_{1}}^{*}=\beta_{k_{1}}+\mu, \quad \beta_{j}^{*}=\beta_{j}, j \neq k_{1} ;
$$

\footnotetext{
†It turns out that the first associated of Jacobi polynomials with parameters $(\alpha, \beta) \in$ $\{(1 / 2,1 / 2),(-1 / 2,1 / 2),(1 / 2,-1 / 2)\}$ are also semi-classical (see the discussion on second degree forms in Section 1.4)
} 
the generalized co-dilated polynomials, where it is modified, at level $k_{2}$, the coefficient $\gamma_{k_{2}}$,

$$
\hat{\gamma}_{k_{2}}=\lambda \gamma_{k_{2}}, \quad \hat{\gamma}_{j}=\gamma_{j}, j \neq k_{2}, \quad \lambda \neq 0 ;
$$

the generalized co-modified polynomials, combining the two previous transformations, with two modifications, $\mu$ and $\lambda$, at levels $k_{1}$ and $k_{2}$. The explicit formulae (17) for these transformations are given in [28, Sec.4].

Generic rational spectral transformations of Stieltjes functions (17) induce polynomial transformations, say $\left\{\tilde{P}_{n}\right\}_{n \geq 0}$, in the form

$$
\tilde{P}_{n}(x)=r_{n}(x) P_{n}(x)+s_{n}(x) P_{n-1}(x)+t_{n}(x) P_{n}^{(1)}(x)+v_{n}(x) P_{n-1}^{(1)}(x),
$$

where $r_{n}, s_{n}, t_{n}, v_{n}$ are rational functions of degrees not depending on $n$. This amounts to the study of polynomial transformations through relations such as

$$
E(x) \tilde{P}_{n}(x)=\sum_{k=n-J}^{n+K} \xi_{n, k} P_{k}(x),
$$

where $E$ is a polynomial of fixed degree, and $J, K$ are positive integers (see [60] and [90, Secs. 3 and 4]). For the generalized co-recursive polynomials, generalized co-dilated polynomials, and generalized co-modified polynomials, explicit formulae of type (18) are given in [28]. A more general case was analysed in [66], where, under certain conditions, it is given closed expressions for the polynomials $\tilde{P}_{n}$ related to $\tilde{S}$ in terms of $P_{n}$ and its associated $P_{n-1}^{(1)}$.

The Laguerre-Hahn character of orthogonal polynomials is invariant under generic rational spectral transformations (17) of Stieltjes functions [90, Prop. 4]. The analysis of such a topic for some of the above referred transformations can be found in [28, 32] (see also [22]). The analysis of transformations on linear functionals can be found in [3, Chap. IV], [30, 31, 33, 69], and [68, Chap. III].

In general, rational spectral transformations of type (17) with $c \not \equiv 0$ produce Laguerre-Hahn orthogonal polynomials whenever applied to Stieltjes functions related to semi-classical polynomials. It remains an open problem to describe all the Laguerre-Hahn orthogonal polynomials in terms of semi-classical ones.

1.4. Introductory remarks and notations. Throughout the text, the Riccati equation (1), $A S^{\prime}=B S^{2}+C S+D$, will be taken in its irreducible form, that is, the polynomials $A, B, C, D$ are co-prime. 
In some situations, the Riccati equation may simplify, with the LaguerreHahn polynomials related to (1) becoming semi-classical. For instance, when $B \equiv 0$, we get the semi-classical case, as already mentioned. But it may happen that the Laguerre-Hahn orthogonal polynomials related to (1) are semi-classical, even with the polynomial $B$ not being identically zero. This is the case of the Stieltjes functions related to second degree forms [59].

A regular linear functional $u$ is called a second degree form if the corresponding Stieltjes function satisfies a second degree equation with polynomial coefficients,

$$
b S^{2}+c S+d=0
$$

where $b, c, d$ satisfy $b \neq 0, c^{2}-4 b d \neq 0, d \neq 0$, due to regularity of $u$. The polynomial $d$ is given in terms of $b, c$ as $d(x)=-\left(u \theta_{0} c\right)(x)+\left(u^{2} \theta_{0}^{2} b\right)(x)$. A second degree form is semi-classical [59, Prop. 2.2]. A necessary and sufficient condition for a Laguerre-Hahn Stieltjes function, satisfying (1), to be related to a second degree form, satisfying (19), is the existence of a polynomial, $p$, such that (see [59] and [68, Chap. IV])

$$
\begin{aligned}
& c^{2}-4 b d=p A, \\
& -2 b\left(b^{\prime} d-b d^{\prime}\right)-c\left(b c^{\prime}-b^{\prime} c\right)=p(c B-b C), \\
& \left.-2 d\left(b c^{\prime}-b^{\prime} c\right)+c\left(b d^{\prime}-b^{\prime} d\right)=p(d B-b D)\right), \\
& d=-\left(u \theta_{0} c\right)+\left(u^{2} \theta_{0}^{2} b\right) .
\end{aligned}
$$

There are some examples where the Stieltjes function satisfies (1) and also (19), for instance, when the associated polynomials of a certain semi-classical family of orthogonal polynomials coincides with the polynomials themselves. This happens with the sequence of Chebyshev polynomials of the second kind, that is, the Jacobi polynomials with parameters $\alpha=\beta=1 / 2$, here denoted by $\left\{P_{n}^{(1 / 2,1 / 2)}\right\}_{n \geq 0}$, as they coincide with the associated polynomials, $\left(P_{n}^{(1 / 2,1 / 2)}\right)^{(1)}=P_{n}^{(1 / 2,1 / 2)}, n \geq 0$; it also holds for the sequence of associated Jacobi polynomials with $\alpha=-1 / 2, \beta=1 / 2$, as we have $\left(P_{n}^{(-1 / 2,1 / 2)}\right)^{(1)}=$ $P_{n}^{(1 / 2,1 / 2)}, n \geq 0$, as well as for the sequence of associated Jacobi polynomials with $\alpha=1 / 2, \beta=-1 / 2$, as we have $\left(P_{n}^{(1 / 2,-1 / 2)}\right)^{(1)}=P_{n}^{(1 / 2,1 / 2)}, n \geq 0$ (see [59] and [68, Chap. IV]). 
The present paper is mainly concerned with analytical aspects of the Laguerre-Hahn theory, leaving the topics on applications for the interested reader. For that purpose, we refer to the papers $[49,51,78]$ and references therein.

The reminder of the paper is organized as follows:

- Section 2 is devoted to the study of differential systems for LaguerreHahn orthogonal polynomials, showing connections between the theory of Laguerre-Hahn orthogonal polynomials and the theory of matrix Riccati equations;

- Section 3 concerns the fourth order differential equations for Laguerre-Hahn orthogonal polynomials;

- Section 4 concerns the classification of Laguerre-Hahn orthogonal polynomials, namely: the so-called Laguerre-Freud equations for recurrence coefficients of Laguerre-Hahn orthogonal polynomials, the class of Laguerre-Hahn linear functionals, the description of some classes (class $s=0$, the symmetric class $s=1$, and the symmetric class $s=2$ ), and recent connections with discrete Painlevé equations;

- Section 5 is devoted to the study of deformed Laguerre-Hahn orthogonal polynomials, that is, the study of evolution of the polynomials and the recurrence coefficients, under some parameter dependence on the polynomials $A, B, C, D$ in the Riccati equation;

- Section 6 contains a brief comment on the Laguerre-Hah class for other types of orthogonality.

There is an Appendix containing two tables with information on the classical orthogonal polynomials.

Before proceeding, some comments on notations and terminology. Throughout the present work, it will be used the notations contained in some of the author's papers, for instance, $[14,15,39]$, as well as in others references, such as $[25,28,90]$. In some references, mainly from P. Maroni and co-authors, e.g., $[29,34,61]$, the Stieltjes function is given as $S(x)=-\sum_{n \geq 0} u_{n} x^{-n-1}$. Thus, our notations differ from theirs on a minus sign in the polynomials $B$ and $D$ in the Riccati equation $A S^{\prime}=B S^{2}+C S+D$, with obvious consequences in some of the related quantities and expressions. 


\section{Differential systems for Laguerre-Hahn orthogonal polynomials}

2.1. From the structure relation to matrix Sylvester differential equations. The structure relation for Laguerre-Hahn orthogonal polynomials was established in [29, Chap. IV, Th. 1.1]. In our notation, it reads as follows.

Theorem 1. Let $u$ be a linear form on $\mathbb{P}$ with values on $\mathbb{C}$, and let $\left\{P_{n}\right\}_{n \geq 0}$ be its SMOP. The following statements are equivalent:

(a) $u$ is a Laguerre-Hahn form satisfying $\mathcal{D}(A u)=\psi u+B\left(x^{-1} u^{2}\right)$;

(b) the sequence $\left\{P_{n}\right\}_{n \geq 0}$ satisfies

$$
A P_{n+1}^{\prime}=-B P_{n}^{(1)}+\sum_{\mu=n-e+1}^{n+l} \theta_{n, \mu} P_{\mu}, \quad n \geq e,
$$

with $l=\max \{t, b\}, e=\max \{s+1, b-1\}, t=\operatorname{deg}(A), b=\operatorname{deg}(B), s=$ $\max \{p, q-1\}-1, p=\operatorname{deg}(\psi), q=\operatorname{deg}(x \psi-A)$.

When $B \equiv 0$ we obtain the structure relations for semi-classical orthogonal polynomials (see [60, Sec. 7] and the references therein).

Equation (20) leads to, in our notation,

$$
\begin{gathered}
A P_{n+1}^{\prime}=\left(l_{n}-C / 2\right) P_{n+1}-B P_{n}^{(1)}+\Theta_{n} P_{n}, \quad n \geq 0, \\
A\left(P_{n}^{(1)}\right)^{\prime}=D P_{n+1}+\left(l_{n}+C / 2\right) P_{n}^{(1)}+\Theta_{n} P_{n-1}^{(1)}, \quad n \geq 0,
\end{gathered}
$$

where, $l_{n}, \Theta_{n}$ are polynomials whose degree is uniformly bounded (see [29, Eqs. (3.9)-(3.10)]). Using the three-term recurrence relation for $\left\{P_{n}\right\}_{n \geq 0}$ as well as for $\left\{P_{n}^{(1)}\right\}_{n \geq 0}$, from (21)-(22) we also get (see [29, Eqs. (3.11)-(3.12)]), for all $n \geq 0$,

$$
\begin{gathered}
A P_{n}^{\prime}=\left(l_{n-1}+\left(x-\beta_{n}\right) \frac{\Theta_{n-1}}{\gamma_{n}}-C / 2\right) P_{n}-B P_{n-1}^{(1)}-\frac{\Theta_{n-1}}{\gamma_{n}} P_{n+1}, \\
A\left(P_{n-1}^{(1)}\right)^{\prime}=\left(l_{n-1}+\left(x-\beta_{n}\right) \frac{\Theta_{n-1}}{\gamma_{n}}+C / 2\right) P_{n-1}^{(1)}+\frac{\Theta_{n-1}}{\gamma_{n}} P_{n}^{(1)}+D P_{n} .
\end{gathered}
$$

Now, equations (21)-(24) can be collected into a differential system for $Y_{n}=$ $\left[\begin{array}{cc}P_{n+1} & P_{n}^{(1)} \\ P_{n} & P_{n-1}^{(1)}\end{array}\right]$ that can be put in the form of a matrix Sylvester equation $[15$, 
Eq. (13)],

$$
A Y_{n}^{\prime}=\mathcal{B}_{n} Y_{n}-Y_{n} \mathcal{C}, \quad n \geq 0,
$$

with the matrices $\mathcal{B}_{n}$ and $\mathcal{C}$ defined by

$$
\begin{gathered}
\mathcal{B}_{n}=\left[\begin{array}{cc}
l_{n} & \Theta_{n} \\
-\frac{\Theta_{n-1}}{\gamma_{n}} & l_{n-1}+\left(x-\beta_{n}\right) \frac{\Theta_{n-1}}{\gamma_{n}}
\end{array}\right], n \geq 1, \mathcal{B}_{0}=\left[\begin{array}{cc}
l_{0} & \Theta_{0} \\
-\Theta_{-1} & l_{-1}+\left(x-\beta_{0}\right) \Theta_{-1}
\end{array}\right], \\
\mathcal{C}=\left[\begin{array}{cc}
C / 2 & -D \\
B & -C / 2
\end{array}\right] .
\end{gathered}
$$

Recalling that matrix Sylvester equations are instances of matrix Riccati equations, then equation (25) provides the link between the Laguerre-Hahn theory of orthogonal polynomials and the theory of matrix Riccati equations $[1]$.

Proceeding with the matrix notation, it will be useful to write the recurrence relations for $\left\{P_{n}\right\}_{n \geq 0}$ and $\left\{P_{n}^{(1)}\right\}_{n \geq 0},(9)$ and (12), respectively, as

$$
Y_{n}=\mathcal{A}_{n} Y_{n-1}, \quad \mathcal{A}_{n}=\left[\begin{array}{cc}
x-\beta_{n} & -\gamma_{n} \\
1 & 0
\end{array}\right], \quad n \geq 1, \quad Y_{0}=\left[\begin{array}{cc}
x-\beta_{0} & 1 \\
1 & 0
\end{array}\right] .
$$

The matrices $\mathcal{A}_{n}$ are commonly called transfer matrices. Let us also introduce one more object, to be used in the sequel. Given the SMOP $\left\{P_{n}\right\}_{n \geq 0}, S$ the corresponding Stieltjes function, and $\left\{P_{n}^{(1)}\right\}_{n \geq 0}$ the sequence of associated polynomials, one defines the sequence of functions of the second kind (see $[86])$,

$$
q_{n+1}=P_{n+1} S-P_{n}^{(1)}, \quad n \geq 0, q_{0}=S .
$$

When $S$ is given through (5), then we have the integral representation, for all $n \geq 0$,

$$
q_{n}(x)=\int_{I} \frac{P_{n}(y)}{x-y} d \mu(y), \quad x \in \mathbb{C} \backslash I .
$$

Due to (9) and (12), $\left\{q_{n}\right\}_{n \geq 0}$ satisfies the recurrence relation

$$
q_{n+1}(x)=\left(x-\beta_{n}\right) q_{n}(x)-\gamma_{n} q_{n-1}(x), \quad n=0,1,2, \ldots,
$$

with initial conditions $q_{-1}=0, q_{0}=S$.

With the above notations in mind, it was established in [15] the following characterization. Hereafter, $I$ denotes the identity matrix. 
Theorem 2. Under the previous notations, the following statements are equivalent.

(a) S satisfies

$$
A S^{\prime}=B S^{2}+C S+D, \quad A, B, C, D \in \mathbb{P}
$$

(b) $Y_{n}$ satisfies the matrix Sylvester equation (25),

$$
A Y_{n}^{\prime}=\mathcal{B}_{n} Y_{n}-Y_{n} \mathcal{C}, \quad n \geq 0,
$$

with the matrices $\mathcal{B}_{n}$ and $\mathcal{C}$ defined in (26)-(27), with $l_{n}, \Theta_{n}$ polynomials of uniformly bounded degrees, satisfying the initial conditions

$\Theta_{0}=A+B-\left(x-\beta_{0}\right)\left(l_{0}-C / 2\right), \quad l_{0}=-\left(x-\beta_{0}\right) D-C / 2, \quad \Theta_{-1}=D, \quad l_{-1}=C / 2 ;$

(c) the transfer matrices $\mathcal{A}_{n}$ satisfy

$$
A \mathcal{A}_{n}^{\prime}=\mathcal{B}_{n} \mathcal{A}_{n}-\mathcal{A}_{n} \mathcal{B}_{n-1}, \quad n \geq 1
$$

(d) $Q_{n}:=\left[\begin{array}{c}q_{n+1} \\ q_{n}\end{array}\right], n \geq 0$, satisfies

$$
A Q_{n}^{\prime}=\left(\mathcal{B}_{n}+(B S+C / 2) I\right) Q_{n}, \quad n \geq 0 .
$$

The matrices $\mathcal{B}_{n}$ satisfy fundamental properties:

$$
\begin{gathered}
\operatorname{tr}\left(\mathcal{B}_{n}\right)=0, \quad n \geq 0 \\
\operatorname{det} \mathcal{B}_{n}=\operatorname{det} \mathcal{B}_{0}+A \sum_{k=1}^{n} \frac{\Theta_{k-1}}{\gamma_{k}}, \quad n \geq 1,
\end{gathered}
$$

with $\operatorname{det} \mathcal{B}_{0}=D(A+B)-(C / 2)^{2}$.

Remark . Relations (32) and (33) play a fundamental role in [36, 37, 39], to derive discrete Painlevé equations for the recurrence coefficients of LaguerreHahn orthogonal polynomials (see more details in Subsection 4.2.2).

2.1.1. Differential systems for semi-classical orthogonal polynomials. Using the previous equations with $B \equiv 0$ in (31), that is,

$$
\begin{gathered}
A q_{n+1}^{\prime}=\left(l_{n}+C / 2\right) q_{n+1}+\Theta_{n} q_{n}, \\
A q_{n}^{\prime}=\left(l_{n-1}+\left(x-\beta_{n}\right) \frac{\Theta_{n-1}}{\gamma_{n}}+C / 2\right) q_{n}-\frac{\Theta_{n-1}}{\gamma_{n}} q_{n+1},
\end{gathered}
$$

combined with the Pearson equation for the weight, $w^{\prime} / w=C / A$, we obtain differential equations for $q_{n} / w$ and $q_{n+1} / w$, as given in the systems (34) below. Alternative derivations of these systems can be found, for instance, in [54, 
Sec. 3]. There, it is used the so-called Laguerre method (see [47, 50, 67]), that is, starting with (29) and using the asymptotic expansion

$$
q_{n}(x)=x^{-n-1}+\mathcal{O}\left(x^{-n-2}\right), \quad x \rightarrow \infty
$$

combined with the differential equation $A S^{\prime}=C S+D$ for the Stieltjes function.

The following characterization holds (see [15] and [54, Sec. 3]).

Theorem 3. Let $\left\{P_{n}\right\}_{n \geq 0}$ be a SMOP with respect to a weight $w$, and let $\left\{q_{n}\right\}_{n \geq 0}$ be the corresponding sequence of functions of the second kind. The weight $w$ is semi-classical and satisfies $w^{\prime} / w=C / A$ if, and only if, $\widetilde{Y}_{n}:=$ $\left[\begin{array}{cc}P_{n+1} & q_{n+1} / w \\ P_{n} & q_{n} / w\end{array}\right]$ satisfies the matrix differential equation

$$
A \widetilde{Y}_{n}^{\prime}=\left(\mathcal{B}_{n}-\frac{C}{2} I\right) \widetilde{Y}_{n}, \quad n \geq 1
$$

where $\mathcal{B}_{n}$ is the matrix associated with the equation $A S^{\prime}=C S+D$ for the Stieltjes transform of $w$.

\subsection{Further results within the theory of matrix Riccati equations.}

A fundamental result in the theory of matrix Riccati equations is Radon's Lemma (see [1] and [70]). For the particular case of matrix Sylvester equations, it reads as follows.

Lemma 1. Let $A$ be a polynomial, let $\mathcal{B}_{n} / A, n \geq 1$, and $\mathcal{C} / A$ be matrices whose entries are integrable functions in a domain $G$ of the complex plane, and let $x_{0} \in G$. If the matrices $\mathcal{P}_{n}$ and $\mathcal{L}, \mathcal{L}$ nonsingular, satisfy

$$
\left\{\begin{array}{l}
A \mathcal{L}^{\prime}=\mathcal{C} \mathcal{L} \\
\mathcal{L}\left(x_{0}\right)=I
\end{array}\right.
$$

and

$$
\left\{\begin{array}{l}
A \mathcal{P}_{n}^{\prime}=\mathcal{B}_{n} \mathcal{P}_{n} \\
\mathcal{P}_{n}\left(x_{0}\right)=Y_{n}\left(x_{0}\right), \quad n \geq 1
\end{array}\right.
$$

then the solution of $A Y_{n}^{\prime}=\mathcal{B}_{n} Y_{n}-Y_{n} \mathcal{C}$, in $G$, is given by

$$
Y_{n}=\mathcal{P}_{n} \mathcal{L}^{-1}, \quad n \geq 1 .
$$

Taking into account the results from Theorem 2, the lemma above can be used to find a representation for the matrices $Y_{n}$ related to $A S^{\prime}=B S^{2}+$ $C S+D$. Note that the representation (37) depends on the solution of two 
matrix linear systems, (35) and (36), which are not always trivially solved. Let us start with some remarks on the solution of (36). A more detailed analysis is done in [15, Sec. 4]. Taking into account Theorem 3 above, in [15] it was searched for a solution of (36) given in terms of some semi-classical $\operatorname{SMOP}\left\{\tilde{P}_{n}\right\}_{n \geq 0}$ as

$$
\mathcal{P}_{n}=e^{\int_{x_{1}}^{x} \frac{\tilde{C}(t)}{2 A(t)} d t} \widetilde{Y}_{n-n_{0}}
$$

where $\widetilde{Y}_{n}$ is defined by

$$
\tilde{Y}_{n}=\left[\begin{array}{cc}
\tilde{P}_{n+1} & \tilde{q}_{n+1} / \tilde{w} \\
\tilde{P}_{n} & \tilde{q}_{n} / \tilde{w}
\end{array}\right]
$$

with $\left\{\tilde{P}_{n}\right\}_{n \geq 0}$ a SMOP with respect to a weight function $\tilde{w}$ and $\left\{\tilde{q}_{n}\right\}_{n \geq 0}$ the corresponding sequence of functions of the second kind. Here, $x_{1}$ may be taken as some complex number, and $\tilde{C}$ is a polynomial such that $\int_{x_{1}}^{x} \frac{\tilde{C}(t)}{2 A(t)} d t$ is defined in suitable domains. Using $\mathcal{P}_{n}$ given by (38) into $A \mathcal{P}_{n}^{\prime}=\mathcal{B}_{n} \mathcal{P}_{n}$, we get $A \widetilde{Y}_{n-n_{0}}^{\prime}=\left(\mathcal{B}_{n}-\tilde{C} / 2 I\right) \widetilde{Y}_{n-n_{0}}$, or, with the shifted indexes $\nu=n-n_{0}$,

$$
A \widetilde{Y}_{\nu}^{\prime}=\left(\mathcal{B}_{\nu+n_{0}}-\tilde{C} / 2 I\right) \widetilde{Y}_{\nu} .
$$

Essentially, the equation above tells us that the sequence $\left\{\widetilde{Y}_{n}\right\}_{n \geq 0}$ is obtained through some shift from a semi-classical SMOP. The complete statement is given in the next lemma [15, Lemma 3].

Lemma 2. Let $S$ be a Stieltjes function that satisfies the Riccati differential equation $A S^{\prime}=B S^{2}+C S+D$. Let $\left\{P_{n}\right\}_{n \geq 0}$ be the corresponding SMOP such that the equations (25), $A Y_{n}^{\prime}=\mathcal{B}_{n} Y_{n}-Y_{n} \mathcal{C}$, hold, with the corresponding system (36). Let the following assumption hold:

$$
\exists \tilde{C} \in \mathbb{P}, \exists n_{0} \geq 1: \mathcal{P}_{n}=e^{\int_{x_{1}}^{x} \frac{\tilde{C}(t)}{2 A(t)} d t} \widetilde{Y}_{n-n_{0}}, \quad n \geq n_{0}+1,
$$

with the $\tilde{Y}_{n}$ 's given as in (39), related to a SMOP $\left\{\tilde{P}_{n}\right\}_{n \geq 0}$. Denote by $\tilde{S}$ the Stieltjes function related to $\left\{\tilde{P}_{n}\right\}_{n \geq 0}$. Then, the following statements hold: (a) $\tilde{S}$ and $S$ are related by a spectral rational transformation,

$$
\tilde{S}=\frac{a+b S}{c+d S}, a, b, c, d \in \mathbb{P}, a d-b c \neq 0
$$

(b) $\tilde{S}$ satisfies

$$
A \tilde{S}^{\prime}=\tilde{C} \tilde{S}+\tilde{D}, \quad \tilde{D} \in \mathbb{P}
$$


the polynomials $\tilde{C}, \tilde{D}$ being related to $B, C, D$ by

$$
\begin{aligned}
(b c-a d) B & =\left(b d^{\prime}-b^{\prime} d\right) A+b d \tilde{C}+d^{2} \tilde{D}, \\
(b c-a d) C & =\left(b c^{\prime}+a d^{\prime}-b^{\prime} c-a^{\prime} d\right) A+(a d+b c) \tilde{C}+2 c d \tilde{D}, \\
(b c-a d) D & =\left(a c^{\prime}-a^{\prime} c\right) A+a c \tilde{C}+c^{2} \tilde{D} .
\end{aligned}
$$

As a consequence of the previous lemmas, we obtain the representation for Laguerre-Hahn orthogonal polynomials given in the theorem that follows (see [15, Th. 4]).

Theorem 4. Let $S$ be a Stieltjes function satisfying $A S^{\prime}=B S^{2}+C S+D$. To $A S^{\prime}=B S^{2}+C S+D$ associate $A Y_{n}^{\prime}=\mathcal{B}_{n} Y_{n}-Y_{n} \mathcal{C}$ and the corresponding systems (35) and (36), $A \mathcal{L}^{\prime}=\mathcal{C} \mathcal{L}, A \mathcal{P}_{n}^{\prime}=\mathcal{B}_{n} \mathcal{P}_{n}$. Let $G$ be a domain in the complex plane such that the entries of the matrices $\mathcal{B}_{n} / A$ and $\mathcal{C} / A$ are integrable in $G$. Assume assumption (40). Then, there exists a polynomial $\tilde{C}$ (defined by (41)-(43)) and a weight function $\tilde{w}=e^{\int \frac{\tilde{C}}{A}}$ such that the following representation holds in $G$ :

$$
Y_{n}=\left[\begin{array}{cc}
\sqrt{\tilde{w}} \tilde{P}_{n-n_{0}+1} & \tilde{q}_{n-n_{0}+1} / \sqrt{\tilde{w}} \\
\sqrt{\tilde{w}} \tilde{P}_{n-n_{0}} & \tilde{q}_{n-n_{0}} / \sqrt{\tilde{w}}
\end{array}\right] E_{n} \mathcal{L}^{-1}, \quad n \geq n_{0}+1
$$

with $\left\{\tilde{P}_{n}\right\}$ the SMOP with respect to $\tilde{w},\left\{\tilde{q}_{n}\right\}$ the corresponding sequence of functions of the second kind, and $E_{n}=\mathcal{P}_{n}\left(x_{0}\right)^{-1} Y_{n}\left(x_{0}\right), x_{0} \in G$.

Some examples where the theorem above applies were studied in $[15$, Sec. 5.2], giving representations for the anti-associated Hermite, Laguerre, and Jacobi polynomials.

It remains to study the representation (37) for Laguerre-Hahn orthogonal polynomials by solving the two general systems (35) and (36).

\section{Fourth-order differential equations}

The study of orthogonal polynomial sequences that are solutions of differential equations

$$
\sum_{j=0}^{N} A_{j} y^{(j)}=0
$$

where $A_{j}$ are polynomials (that may depend on $n$ ) has a long history in the literature of special functions. We refer the interested reader to the survey 
[35]. It was shown, in [46], that the minimal order of a differential equation (44) having orthogonal polynomial solutions could always be reduced to $N=2$ or $N=4$.

When $N=2$ in (44), with $A_{2}, A_{1}$ not depending on $n$ and $A_{0}=\lambda$, where $\lambda$ is some spectral (eigenvalue) parameter depending on $n$, that is,

$$
A_{2} y^{\prime \prime}+A_{1} y^{\prime}+\lambda y=0,
$$

the classification of sequences of orthogonal polynomial solutions is known: the polynomials must be, up to a linear change of variable, a member of the classical families, that is, the Hermite, Laguerre, Jacobi, and Bessel polynomials (see $[11,35]$ ). The study of the semi-classical (and non-classical) case can be traced back to the works of Laguerre [50] and, on a Padé table setting, $[47,67]$, where it is shown that sequences of orthogonal polynomials related to formal generating functions, say $f$, satisfying $A f^{\prime}=C f+D$, with $A, B, C$ polynomials, satisfy second order differential equations with polynomial coefficients of bounded degree. It is well-known, at least from [45] and [46] (see also [6] and [60, pp. 49]), the equivalence between the semi-classical character of a sequence of orthogonal polynomials and the fact that it satisfies a second order differential equation with polynomial coefficients having bounded degree.

For Laguerre-Hahn orthogonal polynomials, the minimal order of differential equations (44) is four. In [34], the fourth order differential equation is given as a determinant of order five. Thanks to the structure relation (20), in $[29$, Chap. IV] the fourth order differential equation is given in the form of a determinant of order three. More recently, in [14], an interpretation of these results was given through the use of the structure relations (21)-(22) written as

$$
A \Psi_{n}^{\prime}=\mathcal{M}_{n} \Psi_{n}+\Theta_{n} \Psi_{n-1}, \quad n \geq 0, \quad \Psi_{n}=\left[\begin{array}{c}
P_{n+1} \\
P_{n}^{(1)}
\end{array}\right]
$$

where $\mathcal{M}_{n}=\left[\begin{array}{cc}l_{n}-\frac{C}{2} & -B \\ D & l_{n}+\frac{C}{2}\end{array}\right]$. It is proved [14, Th. 2] the equivalence between the Riccati equation $A S^{\prime}=B S^{2}+C S+D$ and the differential equation

$$
\tilde{\mathcal{A}}_{n} \Psi_{n}^{\prime \prime}+\tilde{\mathcal{B}}_{n} \Psi_{n}^{\prime}+\tilde{\mathcal{C}}_{n} \Psi_{n}=0_{2 \times 1}, \quad n \geq 1
$$


where $\tilde{\mathcal{A}}_{n}, \tilde{\mathcal{B}}_{n}, \tilde{\mathcal{C}}_{n}$ are matrices given by

$$
\begin{aligned}
& \tilde{\mathcal{A}}_{n}=A^{2} \Theta_{n} I \\
& \tilde{\mathcal{B}}_{n}=A \Theta_{n}\left(A^{\prime} I-\mathcal{M}_{n}-\mathcal{M}_{n-1}\right)-A \Theta_{n-1} \Theta_{n} \frac{\left(x-\beta_{n}\right)}{\gamma_{n}} I-A^{2} \Theta_{n}^{\prime} I, \\
& \tilde{\mathcal{C}}_{n}=\Theta_{n}\left(\frac{\Theta_{n-1} \Theta_{n}}{\gamma_{n}} I-A \mathcal{M}_{n}^{\prime}\right)+\left\{\Theta_{n}\left(\mathcal{M}_{n-1}+\frac{\left(x-\beta_{n}\right)}{\gamma_{n}} \Theta_{n-1} I\right)+A \Theta_{n}^{\prime} I\right\} \mathcal{M}_{n} .
\end{aligned}
$$

The fourth order differential equation is then obtained by taking derivatives in (45). The detailed proof is given in [14, Cor. 3]. A brief outline follows. Note the first and second derivatives of (45),

$$
\begin{aligned}
& \tilde{\mathcal{A}}_{n} \Psi_{n}^{\prime \prime \prime}+\mathcal{I}_{n} \Psi_{n}^{\prime \prime}+\mathcal{J}_{n} \Psi_{n}^{\prime}+\mathcal{K}_{n} \Psi_{n}=0_{2 \times 1}, \\
& \tilde{\mathcal{A}}_{n} \Psi_{n}^{(4)}+\mathcal{E}_{n} \Psi_{n}^{\prime \prime \prime}+\mathcal{F}_{n} \Psi_{n}^{\prime \prime}+\mathcal{G}_{n} \Psi_{n}^{\prime}+\mathcal{H}_{n} \Psi_{n}=0_{2 \times 1},
\end{aligned}
$$

with

$$
\begin{gathered}
\mathcal{I}_{n}=\tilde{\mathcal{A}}_{n}^{\prime}+\tilde{\mathcal{B}}_{n}, \mathcal{\mathcal { J }}_{n}=\tilde{\mathcal{B}}_{n}^{\prime}+\tilde{\mathcal{C}}_{n}, \mathcal{K}_{n}=\tilde{\mathcal{C}}_{n}^{\prime} \\
\mathcal{E}_{n}=2 \tilde{\mathcal{A}}_{n}^{\prime}+\tilde{\mathcal{B}}_{n}, \mathcal{F}_{n}=\tilde{\mathcal{A}}_{n}^{\prime \prime}+2 \tilde{\mathcal{B}}_{n}^{\prime}+\tilde{\mathcal{C}}_{n}, \mathcal{G}_{n}=\tilde{\mathcal{B}}_{n}^{\prime \prime}+2 \tilde{\mathcal{C}}_{n}^{\prime}, \mathcal{H}_{n}=\tilde{\mathcal{C}}_{n}^{\prime \prime} .
\end{gathered}
$$

After computations, where suitable elements are eliminated, we have

$$
\begin{aligned}
& \mathbb{L}_{n, 2}\left(P_{n+1}\right)=-\tilde{\mathcal{B}}_{n}^{(1,2)}\left(P_{n}^{(1)}\right)^{\prime}-\tilde{\mathcal{C}}_{n}^{(1,2)} P_{n}^{(1)}, \\
& \widehat{\mathbb{L}}_{n, 3}\left(P_{n+1}\right)=\widehat{U}_{n}\left(P_{n}^{(1)}\right)^{\prime}+\widehat{V}_{n} P_{n}^{(1)}, \\
& \widehat{\widehat{\mathbb{L}}}_{n, 4}\left(P_{n+1}\right)=\widehat{\widehat{U}}_{n}\left(P_{n}^{(1)}\right)^{\prime}+\widehat{\widehat{V}}_{n} P_{n}^{(1)},
\end{aligned}
$$

with

$$
\begin{aligned}
& \mathbb{L}_{n, 2}=A^{2} \Theta_{n} \mathbb{D}^{2}+\tilde{\mathcal{B}}_{n}^{(1,1)} \mathbb{D}+\tilde{\mathcal{C}}_{n}^{(1,1)} \mathbb{I} \\
& \widehat{\mathbb{L}}_{n, 3}=A^{2} \Theta_{n} \mathbb{L}_{n, 3}+\mathcal{I}_{n}^{(1,2)} \tilde{\mathcal{B}}_{n}^{(2,1)} \mathbb{D}+\mathcal{I}_{n}^{(1,2)} \tilde{\mathcal{C}}_{n}^{(2,1)} \mathbb{I}, \\
& \widehat{U}_{n}=\mathcal{I}_{n}^{(1,2)} \tilde{\mathcal{B}}_{n}^{(2,2)}-\mathcal{J}_{n}^{(1,2)} A^{2} \Theta_{n}, \widehat{V}_{n}=\mathcal{I}_{n}^{(1,2)} \tilde{\mathcal{C}}_{n}^{(2,2)}-\mathcal{K}_{n}^{(1,2)} A^{2} \Theta_{n}, \\
& \widehat{\widehat{\mathbb{L}}}_{n, 4}=A^{2} \Theta_{n} \widehat{\mathbb{L}}_{n, 4}+R_{n} \tilde{\mathcal{B}}_{n}^{(2,1)} \mathbb{D}+R_{n} \tilde{\mathcal{C}}_{n}^{(2,1)} \mathbb{I} \\
& \widehat{\widehat{U}}_{n}=-R_{n} \tilde{\mathcal{B}}_{n}^{(2,2)}+S_{n} A^{2} \Theta_{n}, \widehat{\widehat{V}}_{n}=-R_{n} \tilde{\mathcal{C}}_{n}^{(2,2)}+T_{n} A^{2} \Theta_{n}, \\
& \widehat{\mathbb{L}}_{n, 4}=A^{2} \Theta_{n} \mathbb{L}_{n, 4}-\mathcal{E}_{n}^{(1,2)} \mathcal{I}_{n}^{(2,1)} \mathbb{D}^{2}-\mathcal{E}_{n}^{(1,2)} \mathcal{J}_{n}^{(2,1)} \mathbb{D}-\mathcal{E}_{n}^{(1,2)} \mathcal{K}_{n}^{(2,1)} \mathbb{I}, \\
& R_{n}=\mathcal{E}_{n}^{(1,2)} \mathcal{I}_{n}^{(2,2)}-A^{2} \Theta_{n} \mathcal{F}_{n}^{(1,2)} \\
& S_{n}=\mathcal{E}_{n}^{(1,2)} \mathcal{J}_{n}^{(2,2)}-A^{2} \Theta_{n} \mathcal{G}_{n}^{(1,2)}, \\
& T_{n}=\mathcal{E}_{n}^{(1,2)} \mathcal{K}_{n}^{(2,2)}-A^{2} \Theta_{n} \mathcal{H}_{n}^{(1,2)}
\end{aligned}
$$


Here, $\mathbb{I}$ denotes the identity operator, $\mathbb{D}^{k}$ denotes the derivative operator of order $k, k=1,2$, and the superscript $(i, j)$ on a matrix denotes the element in position $(i, j)$. Equations (46), (47) and (48) yield the fourthorder differential equation for $\left\{P_{n}\right\}_{n \geq 0}$, given in the determinantal form

$$
\left|\begin{array}{lll}
\widehat{\mathbb{U}}_{n, 4}\left(P_{n+1}\right) & \widehat{\widehat{U}}_{n} & \widehat{\widehat{V}}_{n} \\
\widehat{\mathbb{L}}_{n, 3}\left(P_{n+1}\right) & \widehat{U}_{n} & \widehat{V}_{n} \\
\mathbb{L}_{n, 2}\left(P_{n+1}\right) & -\tilde{\mathcal{B}}_{n}^{(1,2)} & -\tilde{\mathcal{C}}_{n}^{(1,2)}
\end{array}\right|=0, \quad n \geq 1 .
$$

Proceeding analogously, one can also obtain a fourth order differential equation for $\left\{P_{n}^{(1)}\right\}_{n \geq 0}$. Furthermore, using the relation

$$
P_{n}^{(k)}(x)=\left(x-\beta_{k+1}\right) P_{n-1}^{(k+1)}(x)-\gamma_{k+1} P_{n-2}^{(k+2)}(x), \quad k \geq 1,
$$

one can obtain a fourth order differential equation for $\left\{P_{n}^{(k)}\right\}_{n \geq 0}, k \geq 1$ (see $[9])$.

Further references focusing on the fourth order differential equation for Laguerre-Hahn orthogonal polynomials are [52, 72, 73, 74, 75].

Remark . Note the equation (46) enclosed by (45). After simplifications, where it is used the identity $l_{n}+l_{n-1}=-\left(x-\beta_{n}\right) \frac{\Theta_{n-1}}{\gamma_{n}}$ (cf. (32)), equation (46) reads as

$A^{2} \Theta_{n} P_{n+1}^{\prime \prime}+A\left(A^{\prime} \Theta_{n}+\Theta_{n} C-A \Theta_{n}^{\prime}\right) P_{n+1}^{\prime}+\tilde{\mathcal{C}}_{n}^{(1,1)} P_{n+1}=-2 A B \Theta_{n}\left(P_{n}^{(1)}\right)^{\prime}-\tilde{\mathcal{C}}_{n}^{(1,2)} P_{n}^{(1)}$, where

$$
\begin{aligned}
\tilde{\mathcal{C}}_{n}^{(1,1)} & =\Theta_{n}\left(\frac{\Theta_{n-1}}{\gamma_{n}} \Theta_{n}-A\left(l_{n}-\frac{C}{2}\right)^{\prime}\right) \\
& +\left(\Theta_{n}\left(l_{n-1}-\frac{C}{2}+\left(x-\beta_{n}\right) \frac{\Theta_{n-1}}{\gamma_{n}}\right)+A \Theta_{n}^{\prime}\right)\left(l_{n}-\frac{C}{2}\right)-\Theta_{n} B D \\
\tilde{\mathcal{C}}_{n}^{(1,2)} & =\Theta_{n} A B^{\prime}-\left(\Theta_{n}\left(l_{n-1}-\frac{C}{2}+\left(x-\beta_{n}\right) \frac{\Theta_{n-1}}{\gamma_{n}}\right)+A \Theta_{n}^{\prime}\right) B-\Theta_{n} B\left(l_{n}+\frac{C}{2}\right) .
\end{aligned}
$$

In the semi-classical case, that is, $B \equiv 0$, we obtain the second order differential equation for $\left\{P_{n}\right\}_{n \geq 0}$ (see [60, Sec. 7]),

$$
A^{2} \Theta_{n} P_{n+1}^{\prime \prime}+A\left(A^{\prime} \Theta_{n}+\Theta_{n} C-A \Theta_{n}^{\prime}\right) P_{n+1}^{\prime}+\tilde{\mathcal{C}}_{n}^{(1,1)} P_{n+1}=0, \quad n \geq 1 .
$$




\section{Classification of Laguerre-Hahn orthogonal polynomi- als}

4.1. Laguerre-Freud equations for the recurrence coefficients. The so-called Laguerre-Freud equations are closely related to the direct problem [85]: to deduce information on the recurrence coefficients of sequences of orthogonal polynomials, given the orthogonality measure. Of course, closed formulae are not always possible to obtain, usually one deals with non-linear systems of equations involving the recurrence coefficients (see [8]). In the literature of semi-classical orthogonal polynomials, such systems have been coined as Laguerre-Freud equations, mainly after the works of A. P. Magnus $[56,57]$.

In the Laguerre-Hahn setting, we pose the direct problem as the somewhat more general question: to seek information for the recurrence coefficients, given the polynomials $A, B, C, D$ in the Riccati equation (1), $A S^{\prime}=B S^{2}+$ $C S+D$.

A first hint towards Laguerre-Freud equations for the recurrence coefficients of Laguerre-Hahn orthogonal polynomials satisfying the recurrence relation (9),

$$
P_{n+1}(x)=\left(x-\beta_{n}\right) P_{n}(x)-\gamma_{n} P_{n-1}(x), \quad n=0,1,2, \ldots,
$$

is the differential systems (25). These, combined with the recurrence relation written in the matrix form (28), yield the the compatibility conditions (30), which, in turn, yield difference equations for the $\beta_{n}$ 's and $\gamma_{n}$ 's. In general, one can deduce recurrences in the form $F_{j}\left(\beta_{n}, \beta_{n-1}, \ldots, \beta_{0}, \gamma_{n}, \gamma_{n-1}, \ldots, \gamma_{1}, n, \ldots\right)=$ $0, j=1, \ldots, N$, where $F_{j}$ are non-linear functions in the $\beta_{n}$ 's and $\gamma_{n}$ 's. In the Laguerre-Hahn setting, some of these recurrences have been identified as forms of discrete Painlevé equations in $[39,36,37]$. The derivation of discrete Painlevé equations for the recurrence coefficient of orthogonal polynomials is a well-know topic in the literature of special functions (see, for instance, $[53,54,85]$ and the recent monograph [84]), more information on this topic will be given in Subsection 4.2.2.

Let us note that, under some restrictions on the degrees of $A, B, C, D$ in the Riccati equation, the non-linear systems involving the recurrence coefficients of the orthogonal polynomials may allow simplifications. Indeed, the polynomials $A, B, C, D$ are not arbitrary, the general theory of distributions 
and the asymptotic expansion (3) imply degree restrictions and other connections between them. In this context, it comes out the notion of class of a Laguerre-Hahn linear functional [4], which we describe in the next section.

4.2. The class of a Laguerre-Hahn linear functional. Recall the distributional equation (13) for Laguerre-Hahn linear functionals whose Stieltjes function $S$ satisfies a Riccati equation $A S^{\prime}=B S^{2}+C S+D$,

$$
\mathcal{D}(A u)=\psi u+B\left(x^{-1} u^{2}\right),
$$

together with the operations in the algebraic dual space of $\mathbb{P}$ described in Section 1.2.

Of course, the equation above is not unique, as we can multiply it by an arbitrary polynomial, $\chi$, thus obtaining $\mathcal{D}(\chi A u)=\left(\chi \psi-\chi^{\prime} A\right) u+(\chi B)\left(x^{-1} u^{2}\right)$. To each triplet $(A, \psi, B)$ from (13) one associates the integer

$$
s=\max \{p-1, d-2\},
$$

with $p=\operatorname{deg}(\psi), d=\max \{t, r\}, t=\operatorname{deg}(A), r=\operatorname{deg}(B)$. It was proved in [3] (see also [4, Prop. 2.1]) that the regularity of $u$ implies $s$ non-negative. Thus, there exists a correspondence $u \rightarrow H(u)$ from the set of LaguerreHahn linear functionals into the subsets of the non-negative integers, that outputs $s$ to each $u$ satisfying (13). The minimum element of $H(u)$ is called the class of $u$. When $u$ is of class $s$, the sequence of orthogonal polynomials with respect to $u$ is also said to be of class $s$. For each Laguerre-Hahn linear functional, the triplet $(A, \psi, B)$ that realizes the minimum of $H(u)$ is unique [4, Prop. 2.3].

The following criterion gives the precise value for the class (see [3] and [4, Prop. 2.4]).

Propposition 1. Let the previous notations hold. The linear functional u satisfying the distributional equation (13) is of class $s=\max \{p-1, d-2\}$ if, and only if,

$$
\prod_{c \in \mathcal{Z}}\left\{\left|A^{\prime}(c)-\psi(c)\right|+|B(c)|+\left|\left\langle u,-\theta_{c} \psi+\theta_{c}^{2} A-u \theta_{0} \theta_{c} B\right\rangle\right|\right\} \neq 0,
$$

where $\mathcal{Z}$ denotes the set os zeros of $A$.

There is also a criterion in terms of the Riccati equation for the Stieltjes function related to $u$, as follows (see [3] and [4, Prop. 2.5]). 
Propposition 2. Let the previous notations hold. The Laguerre-Hahn linear functional whose Stieltjes function satisfies $A S^{\prime}=B S^{2}+C S+D$ is of class $s=\max \{p-1, d-2\}$ if, and only if, the polynomials $A, B, C, D$ have no common zeros.

In the symmetric case, that is, when $\beta_{n}=0, n \geq 0$, in the recurrence relation (9) or, equivalently, when the odd moments of the linear functional $u$ are all zero, the following restrictions on the polynomials $A, B, \psi$ hold $[3,4]$.

Propposition 3. Let $u$ be a symmetric Laguerre-Hahn form of class s, satisfying equation (13). The following holds: if $s$ is even, then $A$ and $B$ are even and $\psi$ is odd; if $s$ is odd, then $A$ and $B$ are odd and $\psi$ is even.

In the account of $A S^{\prime}=B S^{2}+C S+D$, with $C=\psi-A^{\prime}$ (cf. (13)) and $D$ given by (14), then, in the symmetric case, we have the following result: if $s$ is even, then $A$ and $B$ are even, $C$ is odd, and $D$ is even; if $s$ is odd, then $A$ and $B$ are odd, $C$ is even, and $D$ is odd.

In order to avoid redundancies, it is considered the concept of displacement. Given $a, b$ complex numbers, the displacement $h_{a} \circ \tau_{b}$ is defined as $\left(h_{a} \circ\right.$ $\left.\tau_{b}\right) p(x)=p(a x-b), p \in \mathbb{P}$. By transposition, it is defined $\tau_{b} \circ h_{a}$ in the algebraic dual space of $\mathbb{P}$ as $\left\langle\left(\tau_{b} \circ h_{a}\right) u, p\right\rangle=\left\langle u,\left(h_{a} \circ \tau_{b}\right) p\right\rangle$. With these definitions in mind, if a linear functional $\tilde{u}$ of class $s$ satisfies

$$
\mathcal{D}(\tilde{A} \tilde{u})=\tilde{\psi} \tilde{u}+\tilde{B}\left(x^{-1} \tilde{u}^{2}\right),
$$

then the linear functional $u=\left(h_{1 / a} \circ \tau_{b}\right) \tilde{u}$ is of class $s$, and it satisfies

$$
\mathcal{D}(A u)=\psi u+B\left(x^{-1} u^{2}\right),
$$

with

$A(x)=a^{-k}\left(h_{a} \circ \tau_{-b}\right) \tilde{A}(x), \psi(x)=a^{1-k}\left(h_{a} \circ \tau_{-b}\right) \tilde{\psi}(x), B(x)=a^{1-k}\left(h_{a} \circ \tau_{-b}\right) \tilde{B}(x)$, where $k=\operatorname{deg}(A)$. Hence, a displacement does not change neither the Laguerre-Hahn character nor the class of a Laguerre-Hahn linear functional [60]. Therefore, we can take canonical functional equations, by re-situating the zeros of $A$ in equation (13). This will be put in evidence in the sequel.

4.2.1. Description of some classes of Laguerre-Hahn linear functionals. So far, several classes of Laguerre-Hahn linear functionals have been described: the class $s=0[12,13]$; the symmetric class $s=1[3,4]$; the symmetric class $s=2$ when $A$ and $B$ vanish at zero [77]. In all these cases, the explicit 
expressions for the recurrence coefficients of the corresponding orthogonal polynomials are stated. The main results follow.

The class $s=0$ [12, 13].

In this situation we have

$$
A(x)=a_{2} x^{2}+a_{1} x+a_{0}, \quad \psi(x)=\psi_{1} x+\psi_{0}, \quad B(x)=b_{2} x^{2}+b_{1} x+b_{0},
$$

with $\left|a_{2}\right|+\left|\psi_{1}\right|+\left|b_{2}\right|>0$.

Several situations are considered, following the guide of canonical cases from the classical families: the analogue of the Hermite polynomials, with $A(x)=1$; the analogue of the Laguerre polynomials, with $A(x)=x$; the analogue of the Jacobi polynomials, with $A(x)=x^{2}-1$; the analogue of the Bessel polynomials, with $A(x)=x^{2}$. The Laguerre-Hahn orthogonal polynomials of class $s=0$ are the Hermite, Laguerre, Jacobi, Bessel (when $B \equiv 0$ ), as well as some of their perturbations: the co-recursive, co-dilated, associated of order $\tau$, co-recursive of associated of order $\tau$, and co-dilated of associated of order $\tau$.

The symmetric class $s=1[3,4]$.

In this situation we have

$$
A(x)=a_{3} x^{3}+a_{1} x, \quad \psi(x)=\psi_{2} x^{2}+\psi_{0}, \quad B(x)=b_{3} x^{3}+b_{1} x,
$$

with $\left|a_{3}\right|+\left|\psi_{2}\right|+\left|b_{3}\right|>0$. The following canonical cases are obtained.

(a) case $A(x)=x$ : the polynomials $\psi, B$ are

$$
\left\{\begin{array}{l}
\psi(x)=\left(\frac{4(1+2 \mu+\tau)}{\lambda}-2\right) x^{2}-(1+2 \mu), \\
B(x)=2\left(1-\frac{1+\tau+2 \mu}{\lambda}\right) x^{3}+(1+2 \mu-\lambda) x,
\end{array}\right.
$$

the recurrence coefficient $\gamma_{n}$ is defined by

$$
\left\{\begin{array}{l}
\gamma_{1}=\frac{1}{2} \lambda \\
\gamma_{n+1}=\frac{1}{2}\left(n+1+\tau+\mu\left(1+(-1)^{n}\right)\right), \quad n \geq 1,
\end{array}\right.
$$

where $\tau \neq-2(n+1), n \geq 0, \quad \tau+2 \mu \neq-2(n+1), n \geq 1$;

(b) case $A(x)=x^{3}$ : the polynomials $\psi, B$ are

$$
\left\{\begin{array}{l}
\psi(x)=\left(2(\nu-1)-\frac{1}{\lambda(\nu+1)}-\frac{4 \mu}{\lambda}\right) x^{2}+2 \mu \\
B(x)=\left(\frac{1}{\lambda}\left(2 \mu+\frac{1}{2 \nu+2}\right)-2 \mu\right) x^{3}+(2(\nu+1) \lambda-2 \mu) x
\end{array}\right.
$$


the recurrence coefficient $\gamma_{n}$ is defined by

$$
\left\{\begin{array}{l}
\gamma_{1}=\lambda \\
\gamma_{n+1}=\frac{1}{4} \frac{\left\{1+\mu\left(2 \nu+3+(-1)^{n}(2 n+2 \nu+1)\right)\right\}}{(n+\nu)(n+\nu+1)}, \quad n \geq 1,
\end{array}\right.
$$

where $1+4 \mu(n+\nu+1) \neq 0, \quad 1-4 \mu n \neq 0, n \geq 1$

(c) case $A(x)=x\left(x^{2}-1\right)$ : the polynomials $\psi, B$ are

$$
\left\{\begin{array}{l}
\psi(x)=\left(\alpha+\beta-3-\frac{2}{\lambda} \frac{\mu(\alpha+\beta)+\alpha \beta}{\alpha+\beta+1}\right) x^{2}+1+2 \mu \\
B(x)=\left(\frac{1}{\lambda} \frac{\mu(\alpha+\beta)+\alpha \beta}{\alpha+\beta+1}+1-\alpha-\beta\right) x^{3}+(\lambda(1+\alpha+\beta)-1-2 \mu) x,
\end{array}\right.
$$

the recurrence coefficient $\gamma_{n}$ is defined by

$$
\left\{\begin{array}{l}
\gamma_{1}=\lambda \\
\gamma_{n+1}=\frac{(n+\alpha)(n+\beta)+\mu(2 n+\alpha+\beta)(-1)^{n}}{(2 n+\alpha+\beta-1)(2 n+\alpha+\beta+1)}, \quad n \geq 1,
\end{array}\right.
$$

where $\alpha+\beta+1 \neq-2 n, n \geq 0, \quad(2 n+\alpha)(2 n+\beta)+\mu(4 n+\alpha+\beta) \neq 0, n \geq 1$, and $(2 n+1+\alpha)(2 n+1+\beta)-\mu(4 n+\alpha+\beta+2) \neq 0, n \geq 0$.

The symmetric class $s=2$ under restrictions [77].

The description of the symmetric Laguerre-Hahn forms of class $s=2$, under the restrictions $A(0)=B(0)=0$, was done in [77], with the main technique based on the quadratic decomposition of the Stieltjes function (see equation (50) below). Essentially, it is proved that $u$ is symmetric Laguerre-Hahn of class $s=2$ with $A(0)=B(0)=0$ if, and only if, there exists a Laguerre-Hahn functional $v$, of class $\tilde{s}=0$, such that

$$
x \sigma u=-\lambda v, \quad \lambda=-u_{2} .
$$

Here, $u_{2}$ denotes the moment of order 2 of $u$, and the functional $\sigma u$ is defined by $\langle\sigma u, p\rangle=\langle u, \sigma p\rangle$, with $\sigma p(x)=p\left(x^{2}\right), \quad p \in \mathbb{P}$. Condition (49) is equivalent to the relation for the corresponding Stieltjes functions, now denoted by $S_{u}$ and $S_{v}$,

$$
S_{v}\left(x^{2}\right)=-x \lambda^{-1} S_{u}(x)-\lambda^{-1} .
$$

Three canonical cases for the polynomial $A$ appear: $A(x)=x^{2}, A(x)=$ $x^{4}$, and $A(x)=x^{2}\left(x^{2}-1\right)$. In the first case, $v$ is the co-recursive of the associated linear functional of order $\tau$ of Laguerre; in the second case, $v$ is the co-recursive of the associated liner functional of order $\tau$ of Bessel; in the third case, of $v$ is a displacement of the co-recursive of the associated linear 
functional of order $\tau$ of Jacobi. Precise formulae for $B, \psi$ are given in Eqs. (48), (56), (57), (65) of [77].

The description of Laguerre-Hahn class $s=1$, as well as the symmetric Laguerre-Hahn class $s=2$ in the general case, that is, with $A(0) B(0) \neq 0$, remain open problems. Thus, so far, no general closed form for the recurrence coefficients of those Laguerre-Hahn orthogonal polynomials is stated. Recently, these classes have been revisited in [36, 37]: in [36], it is given a recursive way to compute $\beta_{n}$ and $\gamma_{n}$ in Laguerre-Hahn class $s=1$,

$$
\beta_{n}=F\left(\beta_{n-1}, \ldots, \beta_{0}, \hat{f}_{n}\right), \quad \gamma_{n+1}=G\left(\gamma_{n}, \ldots, \gamma_{1}, \beta_{n}, \hat{g}_{n}\right), \quad n \geq 1 ;
$$

with $\hat{f}_{n}, \hat{g}_{n}$ explicit expressions given in terms of the coefficients of the Riccati equation; in [37], on the symmetric class $s=2$, it is given a fourth order difference equation for $\gamma_{n}$ [37, Cor. 4],

$$
\gamma_{n+2}=F\left(\gamma_{n+1}, \gamma_{n}, \gamma_{n-1}, \gamma_{n-2}\right), \quad n \geq 2 .
$$

Remark . Taking into account the classification given in [7], combined with the stability of some of the rational spectral transformations in [90, pp. 78], the Laguerre-Hahn class $s=1$ includes linear functionals such as: $\tilde{u}=R(x) u+\sum_{k=1}^{n_{1}} \lambda_{k} \delta\left(x-\xi_{k}\right)$, where $R$ is some function of the type $R(x)=\prod_{k=1}^{n_{0}}\left|x-\alpha_{k}\right|^{\mu_{k}} e^{\tau_{k} / x+\rho_{k} x^{2}}$, with $\alpha_{k}, \mu_{k}, \tau_{k}, \rho_{k}, \xi_{k}$ suitable real numbers, $\delta\left(x-\xi_{k}\right)$ is the Dirac Delta functional at $\xi_{k}$, and $u$ is the linear functional corresponding to the Hermite, Laguerre, Jacobi, and Bessel polynomials (see $[7])$; the associated and anti-associated polynomials related to some limiting cases of $q$-Racah polynomials when $q \rightarrow-1$ (see [7, pp. 264]); the generalized co-dilated with parameter $k=1$ (see [28, Eqs. (5), (21)]) of the previous functionals.

4.2.2. The class $s=1$ and the symmetric class $s=2$ revisited: connections with discrete Painlevé equations. Discrete Painlevé equations (dP) are nonlinear difference equations which have a continuous Painlevé equation as a continuous limit. The discrete Painlevé equations share many features of their continuous counterparts: some can be fitted into degeneration cascades; for others Lax pairs have been established, hierarchies have been computed, and special solutions can be found, as well as Miura and Bäcklund transformations (see, for instance, [27, 42]). There is a standard list, consisting of the earliest derived discrete Painlevé equations, in [42]. More elaborated lists can be found in $[84,85]$. 
As mentioned previously, the identification of difference equations involving the recurrence coefficients of orthogonal polynomials with discrete Painlevé equations is a well-known subject, much analysis has been carried out within the families of orthogonal polynomials related to semi-classical weights (see, for instance, $[53,84,85])$. Unlike the semi-classical case, the Laguerre-Hahn setting falls short on such a study. To the best of the author's knowledge, the derivation of discrete Painlevé equations for the recurrence coefficients of Laguerre-Hahn orthogonal polynomials on the real line is limited to [39, 36,37 , with focus on the classes $s=1$ and $s=2$. The derivation of the referred discrete Painleve equations is purely algebraic, it is based on the use of the equations enclosed in (30), with the identities (32) and (33) playing a fundamental role. Details will not be given here, the interested reader may find the complete proofs in $[39,36,37]$.

The list is given below. Recall that we are taking SMOPs satisfying the three-term recurrence relation (9),

$$
P_{n+1}(x)=\left(x-\beta_{n}\right) P_{n}(x)-\gamma_{n} P_{n-1}(x), \quad n=0,1,2, \ldots,
$$

and the Riccati equation $A S^{\prime}=B S^{2}+C S+D$ for the corresponding Stieltjes function.

Class $s=1: A(x)=a_{3} x^{3}+a_{2} x^{2}+a_{1} x+a_{0}, \quad B(x)=b_{3} x^{3}+b_{2} x^{2}+b_{1} x+b_{0}$,

$$
C(x)=c_{2} x^{2}+c_{1} x+c_{0}, \quad D(x)=d_{1} x+d_{0},
$$

with $d_{1}, d_{0}$ given by $d_{1}=-a_{3}-b_{3}-c_{2}, d_{0}=-\left(2 a_{3}+2 b_{3}+c_{2}\right) \beta_{0}-a_{2}-b_{2}-c_{1}$. Set $\eta=2 b_{3}+c_{2}, \mu=b_{3}\left(\gamma_{1}+\beta_{0}^{2}\right)+b_{2} \beta_{0}+b_{1}+c_{0} / 2, \lambda=2 b_{3} \beta_{0}+2 b_{2}+c_{1}, \xi_{0}=$ $c_{0}^{2} / 4-d_{0} b_{0}$.

$$
\text { Case } A(x)=a_{0}[39, \text { Th. 3.3]: }
$$

the sequences

$$
x_{n}=\eta \beta_{n}+\lambda / 2, \quad z_{n}=\eta^{2}\left(-n a_{0}+\gamma_{1}\left(\eta\left(\beta_{0}+\beta_{1}\right)+\lambda\right)\right)
$$

satisfy the alternative discrete Painlevé equation $d P_{I}$

$$
\frac{z_{n-1}}{x_{n-1}+x_{n}}+\frac{z_{n}}{x_{n}+x_{n+1}}=-x_{n}^{2}+\gamma, \quad \gamma=(\lambda / 2)^{2}-2 \mu \eta, \quad n \geq 1,
$$

with the initial conditions $x_{0}=\eta \beta_{0}+\lambda / 2, x_{1}=\eta \beta_{1}+\lambda / 2$.

$$
\text { Case } A(x)=a_{1} x[39, \text { Th. 3.2]: }
$$


(i) Let $\eta<0$. The sequences

$$
x_{n}=\frac{\sqrt{-\eta}}{\eta \beta_{n}+\lambda}, \quad y_{n}=-\left(\eta \gamma_{n}+n a_{1}+\mu\right)
$$

satisfy the following discrete system of Painlevé equations $d P_{I V}$

$$
x_{n-1} x_{n}=\frac{y_{n}+n a_{1}+\mu}{y_{n}^{2}-\xi_{0}}, \quad y_{n}+y_{n+1}=\frac{1}{x_{n}}\left(\frac{\lambda}{\sqrt{-\eta}}-\frac{1}{x_{n}}\right), \quad n \geq 1,
$$

with the initial conditions $x_{0}=\frac{\sqrt{-\eta}}{\eta \beta_{0}+\lambda}, y_{1}=-\left(\eta \gamma_{1}+a_{1}+\mu\right)$;

(ii) Let $\eta>0$. The sequences

$$
x_{n}=\frac{\sqrt{\eta}}{\eta \beta_{n}+\lambda}, \quad y_{n}=\eta \gamma_{n}+n a_{1}+\mu
$$

satisfy the following discrete system of Painlevé equations $d P_{I V}$

$$
x_{n-1} x_{n}=\frac{y_{n}-n a_{1}-\mu}{y_{n}^{2}-\xi_{0}}, \quad y_{n}+y_{n+1}=\frac{1}{x_{n}}\left(\frac{\lambda}{\sqrt{\eta}}-\frac{1}{x_{n}}\right), \quad n \geq 1,
$$

with the initial conditions $x_{0}=\frac{\sqrt{\eta}}{\eta \beta_{0}+\lambda}, \quad y_{1}=\eta \gamma_{1}+a_{1}+\mu$.

$$
\text { Case } A(x)=x-\alpha[39, \text { Th. 3.4]: }
$$

the sequences

$$
x_{n}=\frac{k_{1} \eta}{\eta \beta_{n}+k_{2}(\eta \alpha+\lambda)}, \quad y_{n}=\frac{k_{2}^{2}\left(\eta \gamma_{n}+n+\mu+g_{1} / 2\right)}{\eta k_{1}^{2}}
$$

satisfy, for all $n \geq 1$,

$x_{n-1} x_{n}=\frac{\eta k_{1}^{2} y_{n}-k_{2}^{2}\left(n+\mu+g_{1} / 2\right)}{\eta k_{1}^{2}\left(y_{n}^{2}-\left(c_{0}^{2} / 4+b_{0}\left(b_{2}+c_{1}+\eta \beta_{0}\right)\right)\right)}, \quad y_{n}+y_{n+1}=\frac{k_{2} x_{n}(\lambda+2 \eta \alpha)-\eta k_{1}}{\eta k_{1} x_{n}^{2}}$.

Here, $g_{1}=\eta \alpha^{2}+\lambda \alpha$, and $k_{1}, k_{2}$ are constants. The initial conditions are given by

$$
x_{0}=\frac{k_{1} \eta}{\eta \beta_{0}+k_{2}(\eta \alpha+\lambda)}, \quad y_{1}=\frac{k_{2}^{2}\left(\eta \gamma_{1}+1+\mu+g_{1} / 2\right)}{\eta k_{1}^{2}} .
$$

Remark . Note that if we take $\alpha=0$ in (57) combined with $k_{1}=\sqrt{\eta} / \eta, k_{2}=$ 1 , then (58) agrees with (56).

Case $A(x)=a_{2}\left(x-\alpha_{1}\right)\left(x+\alpha_{1}\right)$, with $\alpha_{1} \neq 0$ [36, Th. 4.1]: 
the sequences

$$
\begin{gathered}
F_{n}=\mu+a_{2} S_{n}+\eta \gamma_{n+1}+\left((n+1) a_{2}+\lambda / 2\right) \alpha_{1}, \\
G_{n}=\frac{\eta\left(-\alpha_{1}+\beta_{n}\right)+(2 n+1) a_{2}+\lambda}{\eta\left(\alpha_{1}+\beta_{n}\right)+(2 n+1) a_{2}+\lambda},
\end{gathered}
$$

satisfy the following system of equations - a similar form of a $d P_{V}$, for all $n \geq 1$,

$$
\begin{gathered}
\frac{\left(F_{n}-a_{n, 1}\right)\left(F_{n}-b_{n, 1}\right)}{\left(F_{n}-a\right)\left(F_{n}-b\right)}=G_{n+1} G_{n} \\
F_{n}+F_{n-1}=-\alpha_{1}^{2} \eta+\frac{-2 \alpha_{1}\left(2 \alpha_{1} \eta+\lambda+(2 n+1) a_{2}\right)}{G_{n}-1}+\frac{-4 \alpha_{1}^{2} \eta}{\left(G_{n}-1\right)^{2}},
\end{gathered}
$$

with

$$
\begin{gathered}
a_{n, 1}=2\left((n+1) a_{2}+\frac{\lambda}{2}\right) \alpha_{1}-\frac{\eta}{2} \alpha_{1}^{2}+\sqrt{\zeta_{2}}, \quad b_{n, 1}=a_{n, 1}-2 \sqrt{\zeta_{2}} \\
a=-\frac{\eta}{2} \alpha_{1}^{2}+\sqrt{\zeta_{1}}, \quad b=a-2 \sqrt{\zeta_{1}}
\end{gathered}
$$

where $\zeta_{1}=-\left(D(A+B)-(C / 2)^{2}\right)\left(\alpha_{1}\right), \zeta_{2}=-\left(D(A+B)-(C / 2)^{2}\right)\left(-\alpha_{1}\right)$. The initial conditions hold:

$$
F_{0}=\mu+a_{2} \beta_{0}+\eta \gamma_{1}+\left(a_{2}+\lambda / 2\right) \alpha_{1}, \quad G_{1}=\frac{\eta\left(-\alpha_{1}+\beta_{1}\right)+3 a_{2}+\lambda}{\eta\left(\alpha_{1}+\beta_{1}\right)+3 a_{2}+\lambda} .
$$

$\underline{\text { Symmetric class } s=2}: A(x)=a_{4} x^{4}+a_{2} x^{2}+a_{0}, \quad B(x)=b_{4} x^{4}+b_{2} x^{2}+b_{0}$,

$$
C(x)=c_{3} x^{3}+c_{1} x, \quad D(x)=d_{2} x^{2}+d_{0},
$$

with $d_{2}, d_{0}$ given by $d_{2}=-a_{4}-b_{4}-c_{3}, d_{0}=-a_{2}-b_{2}-c_{1}-\gamma_{1}\left(3 a_{4}+2 b 4+c_{3}\right)$. Set $\lambda=\gamma_{1} b_{4}+b_{2}+c_{1} / 2, \mu=2 b_{4}+c_{3}, \tau=b_{4}\left(\gamma_{1}+\gamma_{2}\right) \gamma_{1}+b_{2} \gamma_{1}+b_{0}$.

Case $A(x)=1$ [37, Th. 4]:

the coefficients $\gamma_{n}$ satisfy the discrete Painlevé equation $d P_{I}$

$$
\gamma_{n}\left(\mu\left(\gamma_{n-1}+\gamma_{n}+\gamma_{n+1}\right)+2 \lambda\right)=-n-\tau, \quad n \geq 1,
$$

with $\gamma_{0}=0$ and $\gamma_{1}=u_{2} / u_{0}$, with $u_{j}$ denoting the moment of order $j$ of the linear functional related to $S$.

$$
\text { Case } A(x)=x^{2}+a_{0}[37, \text { Th. } 5] \text { : }
$$


if the following condition holds,

$$
4\left(a_{0} b_{2}-b_{0}\right) d_{0}=a_{0}^{3} \mu^{2}-4 a_{0}^{2} \mu(1+\lambda)-4 a_{0}\left(d_{2}\left(a_{0}+b_{0}\right)-c_{1}^{2} / 4\right),
$$

then the sequence $x_{n}=n+1+\lambda+\mu \gamma_{n+1}-\mu a_{0} / 2$ satisfies the discrete Painlevé equation $d P_{I I}$

$$
\left(x_{n-1}+x_{n}\right)\left(x_{n}+x_{n+1}\right)=\frac{-4 x_{n}^{2}}{\left(\hat{\lambda} x_{n}+z_{n}\right)}, \quad n \geq 1,
$$

with $\hat{\lambda}=\frac{4}{\mu a_{0}}, z_{n}=2-\frac{4(n+1+\lambda)}{\mu a_{0}}$, and initial conditions $x_{0}=1+\lambda+\mu \gamma_{1}-$ $\mu a_{0} / 2, \quad x_{1}=2+\lambda+\mu \gamma_{2}-\mu a_{0} / 2$.

In order to have a complete framework, it is required to deduce discrete Painlevé equations also in the following cases: $A(x)=a_{2} x^{2}, a_{2} \neq 0$, and $A(x)=a_{3} x^{3}+a_{2} x^{2}+a_{1} x+a_{0}, a_{3} \neq 0$, concerning the Laguerre-Hahn class $s=1 ; A(x)=a_{4} x^{4}+a_{2} x^{2}+a_{0}, a_{4} \neq 0$, concerning the Laguerre-Hahn symmetric class $s=2$. It remain open problems.

4.3. Integral representations. The representation of Laguerre-Hahn linear functionals through suitable measures is, in general, an open problem. Nevertheless, there is a class of Laguerre-Hahn linear functionals whose explicit expressions for the measure is known: the linear functionals related to the associated of Hermite, Laguerre and Jacobi polynomials. Explicit formulae are given in [5], [25, Chap. VI], [66] and [88].

Recall that, by virtue of equation (15), the associated polynomials can be viewed as orthogonal polynomials related to spectral transformations of Stieltjes functions such as (17),

$$
\tilde{S}(x)=\frac{a(x) S(x)+b(x)}{c(x) S(x)+d(x)} .
$$

In this setting, we would like to emphasize the results in [66, Th. 3.9], where, given the weight function $w$ related to $S$, being $w$ supported on the interval $[-1,1]$, it is obtained an explicit expression for the weight function related to $\tilde{S}$. As a basic consequence, one can obtain closed expressions for the weight function corresponding to the associated Jacobi polynomials.

Let us also emphasize [86, Sec. 3], where the Stieltjes function of the measure $\mu^{(k)}$ for the $k$ th associated orthogonal polynomials $\left\{P_{n}^{(k)}\right\}_{n \geq 0}$, is given 
in terms of a ratio of functions of the second kind of $\left\{P_{n}\right\}_{n \geq 0}$,

$$
\int \frac{d \mu^{(k)}(y)}{x-y}=\frac{1}{a_{k}} \frac{q_{k}(x)}{q_{k-1}(x)}, \quad x \in \mathbb{C} \backslash \mathbb{R},
$$

where, in our notation, $a_{k}=\sqrt{\gamma_{k}}$, being $\gamma_{k}$ the recurrence coefficient in (9). Recall that the measure $\mu^{(k)}$ can be restored by means of Stieltjes' inversion formula (6). A detailed study of the functions of the second kind of Legendre and Jacobi polynomials can be found in [43, 44].

\section{Deformed Laguerre-Hahn orthogonal polynomials}

The study of deformations on orthogonal polynomials appears in a vast list of papers, mainly focusing on orthogonal polynomials related to semi-classical weights. Usually, the deformation occurs via a $t$-dependence parameter on the weight (in many contexts, $t$ is interpreted as a time variable), and the goal is to study the $t$-evolution of the recurrence coefficients of the orthogonal polynomials under such a dependence. Connections to random matrix theory, Lax pairs, Toda lattices, Painlevé equations, isomonodromic deformations, etc, are well-known and, indeed, intensively studied in the literature (see, amongst many others, [10, 24, 26, 54, 91]). Essentially, two methods of study have emerged: one has been to use a formulation in terms of Lax pairs for isomonodromic deformations of linear differential equations [54], and the other proceeds via the ladder operator technique for orthogonal polynomials [23]. A similar problem to the above described is posed for the Laguerre-Hahn class on the real line in the following terms [17]: given some $t$-dependence on the polynomials $A, B, C, D$ involved in the Riccati equation for the Stieltjes function, $A S^{\prime}=B S^{2}+C S+D$, to study the dynamics of the recurrence coefficients of the orthogonal polynomials related to $S$. To the best of the author's knowledge, so far, such a problem was studied in [17] only. There, the approach is based on some of the techniques in the framework of semiclassical orthogonal polynomials used in [54, Sec. 4]. Here it is a brief outline. In what follows, $\dot{f}$ denotes the derivative of a function $f$ with respect to $t$.

Firstly, recall the equivalence between the Riccati differential equation $A S^{\prime}=B S^{2}+C S+D$ and the matrix Sylvester equations (25),

$$
A Y_{n}^{\prime}=\mathcal{B}_{n} Y_{n}-Y_{n} \mathcal{C}, \quad n \geq 0
$$


for the corresponding $Y_{n}=\left[\begin{array}{cc}P_{n+1} & P_{n}^{(1)} \\ P_{n} & P_{n-1}^{(1)}\end{array}\right]$, with $\left\{P_{n}\right\}_{n \geq 0}$ the SMOP related to $S$, and $\left\{P_{n}^{(1)}\right\}_{n \geq 0}$ the sequence of associated polynomials of the first kind. Now, assuming a $t$-dependence on $A, B, C, D$, define the matrices $\mathcal{H}_{n}$ as

$$
\mathcal{H}_{n}=\left(\dot{Y}_{n}+Y_{n} \dot{\mathcal{L}} \mathcal{L}^{-1}\right) Y_{n}^{-1}, \quad n \geq 1
$$

with $\mathcal{L}$ being a nonsingular matrix such that $A \mathcal{L}^{\prime}=\mathcal{C} \mathcal{L}$. The compatibility between $\frac{\partial \dot{Y}_{n}}{\partial x}$ and $\frac{\partial Y_{n}^{\prime}}{\partial t}$ yields $[17$, Th. 4$]$

$$
\frac{\partial}{\partial t}\left(\frac{\mathcal{B}_{n}}{A}\right)=\mathcal{H}_{n}^{\prime}+\mathcal{H}_{n} \frac{\mathcal{B}_{n}}{A}-\frac{\mathcal{B}_{n}}{A} \mathcal{H}_{n}, \quad n \geq 1 .
$$

Combining (66) with (28) gives us a lax Pair that yields the differential equation for the transfer matrices,

$$
\dot{\mathcal{A}}_{n}=\mathcal{H}_{n} \mathcal{A}_{n}-\mathcal{A}_{n} \mathcal{H}_{n-1}, \quad n \geq 2 .
$$

The differential equations in $t$ involving the recurrence coefficients $\beta_{n}, \gamma_{n}$ can then be obtained via the use of the equations enclosed in (67) and (68).

Let us emphasize the similarity between equations (67) and [54, Eq. 24]. Indeed, formally, these systems are the same, but the matrix $H$ in [54] (cf. [54, pp. 223]), within the semi-classical setting, is different from $\mathcal{H}_{n}$, defined by (66) (here, caution is needed with the notation: the matrix $A$ in $[54$, Eq. 24] should be taken as $\mathcal{B}_{n} / A$ in our notation). Also, it is well to emphasize that $\mathcal{H}_{n}$ in (66) depends on $\mathcal{L}$, which is the solution of a differential system with matrix coefficients having polynomial entries, $A \mathcal{L}^{\prime}=\mathcal{C} \mathcal{L}$. This is a first difficulty when analysing the Laguerre-Hahn case versus the semi-classical case.

Examples of time deformed Laguerre-Hahn orthogonal polynomials, together with differential equations for the recurrence coefficients, can be found in [17], the interested reader is invited to look at [17, Secs. 3.1 and 4].

The general case, that is, how to get general Toda equations for recurrence coefficients of Laguerre-Hahn orthogonal polynomials, of similar type as the ones in [54, Th. 2], via (67) and (68), remains an open problem.

\section{Final remarks}

This paper is focused on the main analytic properties of Laguerre-Hahn orthogonal polynomials on the real line. There are studies on Laguerre-Hahn 
orthogonal polynomials concerning other types of orthogonality, such as discrete orthogonality and orthogonality on the unit circle. These topics require specificities that will not be detailed here. The interested reader may find further information on the following references: [16, 38, 40, 55], concerning discrete orthogonality; $[18,19,20,21,71]$, concerning orthogonality on the unit circle.

\section{Appendix}

The following table gives the weight function as well as the coefficients of the differential equation $A S^{\prime}=C S+D$ for the Stieltjes function related to the classical orthogonal polynomials - the Hermite, Laguerre, Jacobi, and Bessel polynomials (see [60]). These are denoted by $H_{n}, L_{n}^{(\alpha)}, P_{n}^{(\alpha, \beta)}, B_{n}^{(\alpha)}$, respectively. For the Bessel polynomials, we adopt the definition from $[25$, Eq. (4.3), pp. 182].

\begin{tabular}{||l|l|l|l|l||}
\hline \hline & $A$ & $C$ & $D$ & weight \\
\hline$H_{n}$ & 1 & $-2 x$ & 2 & $w(x)=e^{-x^{2}}$ \\
\hline$L_{n}^{(\alpha)}$ & $x$ & $-x+\alpha$ & 1 & $w(x)=x^{\alpha} e^{-x}$ \\
\hline$P_{n}^{(\alpha, \beta)}$ & $x^{2}-1$ & $(\alpha+\beta) x-\beta+\alpha$ & $-1-\alpha-\beta$ & $w(x)=(1-x)^{\alpha}(1+x)^{\beta}$ \\
\hline$B_{n}^{(\alpha)}$ & $x^{2}$ & $\alpha x+2$ & $-1-\alpha$ & $w(x)=x^{\alpha} e^{-2 / x}$ \\
\hline \hline
\end{tabular}

Table 1

The following table gives the recurrence coefficients $\beta_{n}, \gamma_{n+1}, n \geq 0$, in equation (9) for the classical orthogonal polynomials (see [25]).

\begin{tabular}{||l|l|l||}
\hline \hline & $\beta_{n}$ & $\gamma_{n+1}$ \\
\hline$H_{n}$ & 0 & $\frac{n+1}{2}$ \\
\hline$L_{n}^{(\alpha)}$ & $2 n+\alpha+1$ & $(n+1)(n+\alpha+1)$ \\
\hline$P_{n}^{(\alpha, \beta)}$ & $\frac{\beta^{2}-\alpha^{2}}{(2 n+\alpha+\beta)(2 n+\alpha+\beta+2)}$ & $\frac{4(n+1)(n+\alpha+1)(n+\beta+1)(n+\alpha+\beta+1)}{(2 n+\alpha+\beta+1)(2 n+\alpha+\beta+2)^{2}(2 n+\alpha+\beta+3)}$ \\
\hline$B_{n}^{(\alpha)}$ & $\frac{-2 \alpha}{(n+\alpha)(2 n+\alpha+2)}$ & $\frac{-4(n+1)(n+\alpha+1)}{(2 n+\alpha+1)(2 n+\alpha+2)^{2}(2 n+\alpha+3)}$ \\
\hline \hline
\end{tabular}




\section{Table 2}

\section{References}

[1] H. Abou-Kandil, G. Freiling, V. Ionescu, and G. Jank, Matrix Riccati Equations in Control and Systems Theory, Birkhäuser, Basel 2003.

[2] N. I. Akhiezer, The classical moment problem and some related questions in analysis. Translated by N. Kemmer Hafner Publishing Co., New York 1965.

[3] J. Alaya, Quelques résultats nouveaux dans la théorie des polynômes de Laguerre - Hahn, Thèse de Doctorat, Univ. Tunis II, 1996 (in French).

[4] J. Alaya and P. Maroni, Symmetric Laguerre - Hahn forms of class s = 1, Integral Transforms Spec. Funct. 4 (1996), 301-320.

[5] R. Askey and J. Wimp, Associated Laguerre and Hermite polynomials, Proc. Roy. Soc. Edin. 96 (1984), 15-37.

[6] F.V. Atkinson and W.N. Everitt, Orthogonal polynomials which satisfy second order differential equations, in E. B. Christoffel, Birkhäuser, Basel-Boston (1981), pp. 173-181.

[7] S. Belmehdi, On semi-classical linear functionals of class $\mathrm{s}=1$. Classification and integral representations, Indag Math. 3 (1992), 253-275.

[8] S. Belmehdi and A. Ronveaux, Laguerre-Freud's equations for the recurrence coefficients of semi-classical orthogonal polynomials, J. Approx. Theory. 76 (1994), 351-368.

[9] S. Belmehdi and A. Ronveaux, The fourth-order differential equation satisfied by the associated orthogonal polynomials, Rend. Mat. Appl. 11 (1991), 313-326.

[10] P. Bleher and A. Its, Semiclassical asymptotics of orthogonal plynomials, Riemann-Hilbert problem, and universality in the matrix model, Ann. of Math. 150 (1999), 185-266.

[11] S. Bochner, Über Sturm-Liouvillesche polynomsysteme, Math. Zeit. 29 (1929), 730-736.

[12] H. Bouakkaz, Les polynômes orthogonaux de Laguerre-Hahn de classe zéro, Thèse de doctorat, Univ. Pierre et Marie Curie, Paris, 1990 (in French).

[13] H. Bouakkaz and P. Maroni, Description des polynômes orthogonaux de Laguerre-Hahn de classe zéro, in: Orthogonal Polynomials and Their Applications (Erice 1990), IMACS Ann. Comput. Appl. Math. 9, Baltzer, Basel, 1991, pp. 189-194.

[14] A. Branquinho, A. Foulquié Moreno, A. Paiva, and M.N. Rebocho, Second-order differential equations in the Laguerre-Hahn class, Appl. Numer. Math. 94 (2015), 16 - 32.

[15] A. Branquinho, A. Paiva, and M.N. Rebocho, Sylvester equations for Laguerre-Hahn orthogonal polynomials on the real line, Appl. Math. Comput. 219 (2013), 9118-9131.

[16] A. Branquinho and M.N. Rebocho, Characterization theorem for Laguerre-Hahn orthogonal polynomials on non-uniform lattices, J. Math. Anal. Appl. 427 (2015), 185-201.

[17] A. Branquinho and M.N. Rebocho, Deformed Laguerre-Hahn orthogonal polynomials on the real line, Appl. Math. Comput. 233 (2014), 359--368.

[18] A. Branquinho and M.N. Rebocho, Difference and differential equations for deformed Laguerre-Hahn orthogonal polynomials on the unit circle, J. Phys. A 44 (2011), 465204 (12 pp.).

[19] A. Branquinho and M.N. Rebocho, Matrix Sylvester equations in the theory of orthogonal polynomials on the unit circle, Belg. Math. Soc. Simon Stevin 17 (2010), 355-376.

[20] A. Branquinho and M.N. Rebocho, Distributional equation for Laguerre-Hahn functionals on the unit circle, J. Comput. Appl. Math. 233 (2009), 634-642.

[21] A. Branquinho and M.N. Rebocho, On differential equations for orthogonal polynomials on the unit circle, J. Math. Anal. Appl. 356 (2009), 242-256. 
[22] K. Castillo and M.N. Rebocho, On linear spectral transformations and the Laguerre-Hahn class, Integral Transforms Spec. Funct. 28 (2017), 859-875.

[23] Y. Chen and M.E.H. Ismail, Ladder operators and differential equations for orthogonal polynomials. J. Phys. A 30 (1997), 7817--7829.

[24] Y. Chen and A. Its, Painlevé III and a singular linear statistics in Hermitian random matrix ensembles. I, J. Approx. Theory 162 (2010), 270--297.

[25] T.S. Chihara, An Introduction to Orthogonal Polynomials. Gordon and Breach, New York; 1978.

[26] D. V. Chudnovsky, Riemann monodromy problem, isomonodromy deformation equations and completely integrable systems, pp. 385-447 in Bifurcation Phenomena in Mathematical Physics and Related Topics, Proceedings Cargése, 1979 (C. Bardos and D. Bessis , editors), NATO ASI series C, 54, D. Reidel, Dordrecht, 1980.

[27] P.A. Clarkson, Painlevé equations-—nonlinear special functions. Orthogonal polynomials and special functions, 331-411, Lecture Notes in Math., 1883, Springer, Berlin, 2006.

[28] J.S. Dehesa, F. Marcellán, and A. Ronveaux, On orthogonal polynomials with perturbed recurrence relations, J. Comput. Appl. Math. 30 (1990), 203--212.

[29] J. Dini, Sur les formes linéaires et les polynômes orthogonaux de Laguerre-Hahn, Thèse de doctorat, Univ. Pierre et Marie Curie, Paris, 1988 (in French).

[30] J. Dini and P. Maroni, La multiplication d'une forme linéaire par une fraction rationnelle. Application aux formes de Laguerre-Hahn, Ann. Polon. Math. 52 (1990), 175-185.

[31] J. Dini and P. Maroni, The product of a linear form by a rational fraction: application to Laguerre-Hahn forms. Orthogonal polynomials and their applications (Laredo, 1987), 131-138, Lecture Notes in Pure and Appl. Math., 117, Dekker, New York, 1989.

[32] J. Dini, P. Maroni, and A. Ronveaux, Sur une perturbation de la récurrence vérifiée par une suite de polynômes orthogonaux, Portugal. Math. 46 (3) (1989), 269-282.

[33] H. Dueñas, F. Marcellán, and E. Prianes, Perturbations of Laguerre-Hahn functional: modification by the derivative of a Dirac delta, Integral Transforms Spec. Funct. 20 (2009), 59-77.

[34] J. Dzoumba, Sur les polynômes de Laguerre-Hahn, Thèse de doctorat, Univ. Pierre et Marie Curie, Paris, 1985 (in French).

[35] W.N. Everitt and L.L. Littlejohn, Orthogonal polynomials and spectral theory: a survey, in: C. Brezinski, L. Gori, A.A. Ronveaux (Eds.), Orthogonal Polynomials and Their Applications, IMACS Ann. Comput. Appl. Math., vol. 9, J.C. Baltzer AG Publishers, 1991, p. 21-55.

[36] G. Filipuk and M.N. Rebocho, Classification of Laguerre-Hahn orthogonal polynomials of class one, submitted.

[37] G. Filipuk and M.N. Rebocho, The symmetric semi-classical orthogonal polynomials of class two and some of their extensions, submitted (preprint available at http://www.mat.uc.pt/preprints/ps/p1823.pdf)

[38] G. Filipuk and M.N. Rebocho, Orthogonal polynomials on systems of non-uniform lattices from compatibility conditions, J. Math. Anal. Appl. 456 (2017), 1380--1396.

[39] G. Filipuk and M.N. Rebocho, Discrete Painlevé Equations for Recurrence Coefficients of Laguerre-Hahn Orthogonal Polynomials of Class One, Integral Transforms Spec. Funct. 27 (2016), 548-565.

[40] M. Foupouagnigni and F. Marcellán, Characterization of the $D_{w}$-Laguerre-Hahn funtionals, J. Difference Equ. Appl. 8 (2002), no. 8, 689-717.

[41] G. Freud, Orthogonal Polynomials, Pergamon, Oxford, 1971.

[42] B. Grammaticos and A. Ramani, Discrete Painlevé equations: Coalescences, limits and degeneracies, Physica A 228 (1996), 160-171. 
[43] C.C. Grosjean, The weight functions, generating functions and miscellaneous properties of the sequences of orthogonal polynomials of the second kind associated with the Jacobi and the Gegenbauer polynomials, J. Comput. Appl. Math. 16 (1986), 259-307.

[44] C.C. Grosjean, Theory of recursive generation of systems of orthogonal polynomials: An illustrative example, J. Comput. Appl. Math. 12\&13 (1985), 299-318.

[45] W. Hahn, Über Differentialgleichungen für Orthogonalpolynome. (German) [On differential equations for orthogonal polynomials] Monatsh. Math. 95 (1983), 269-274.

[46] W. Hahn, On Differential Equations for Orthogonal Polynomials, Funkcialaj Ek. 21 (1978), $1-9$.

[47] E. Hendriksen and H. van Rossum, A Padé-type approach to non-classical orthogonal polynomials, J. Math. Anal. Appl. 106 (1985), 237--248.

[48] E. Hendriksen and H. Van Rossum, Semiclassical orthogonal polynomials, in: C. Brezinski, A. Draux, A.P. Magnus, P. Maroni, A. Ronveaux (Eds.), Orthogonal Polynomials and Applications (Bar-le-Duc, 1984), Lecture Notes in Math. 1171, Springer Verlag, Berlin, 1985, p. $345-361$.

[49] M.E.H. Ismail, J. Letessier, and G. Valent, Birth and death processes with absorption, Internat. J. Math. Math. Sci. 15 (1992), 469--480.

[50] E.N. Laguerre, Sur la réduction en fractions continues d'une fraction qui satisfait à une équation différentielle linéaire du premier ordre dont les coefficients sont rationnels, J. Math. Pures Appl. 4 (1885), 135--165 = pp. 685-711 in Oeuvres, vol. II, Chelsea, New York 1972.

[51] J. Letessier, Some results on co-recursive associated Laguerre and Jacobi polynomials, SIAM J. Math. Anal. (25) (1994), 528-548.

[52] J. Letessier, On co-recursive associated Laguerre polynomials, J. Comput. Appl. Math. 49 (1993), 127-136.

[53] A.P. Magnus, Freud's equations for orthogonal polynomials as discrete Painlevé equations, Symmetries and integrability of difference equations (Canterbury, 1996). London Mathematical Society Lecture Notes Series, Vol. 255, Cambridge University Press, Cambridge, 1999, pp. $228-243$.

[54] A.P. Magnus, Painlevé-type differential equations for the recurrence coefficients of semiclassical orthogonal polynomials, J. Comput. Appl. Math. 57 (1995), 215-237.

[55] A.P. Magnus, Associated Askey-Wilson polynomials as Laguerre-Hahn orthogonal polynomials, Springer Lect. Notes in Math. 1329, Springer, Berlin, 1988, pp. 261-278.

[56] A.P. Magnus, On Freud's equations for exponential weights, J. Approx. Theory 46(1) (1986), $65-99$.

[57] A.P. Magnus, A proof of Freud's conjecture about the orthogonal polynomials related to $|x|^{\rho} \exp \left(-x^{2 m}\right)$, for integer $m$. Orthogonal polynomials and applications (Bar-le-Duc, 1985), Lecture Notes in Mathematics. Vol. 1171, Springer, Berlin, 1985, pp. 362-372.

[58] A.P. Magnus, Riccati acceleration of Jacobi continued fractions and Laguerre-Hahn orthogonal polynomials. Padé approximation and its applications, Bad Honnef 1983 (Bad Honnef, 1983), 213--230, Lecture Notes in Math., 1071, Springer, Berlin, 1984.

[59] P. Maroni, An introduction to second degree forms. Adv. Comput. Math. 3 (1995), 59--88.

[60] P. Maroni, Une théorie algébrique des polynômes orthogonaux. Application aux polynômes orthogonaux semi-classiques. (Erice, 1990), 95--130, IMACS Ann. Comput. Appl. Math., 9, Baltzer, Basel, 1991.

[61] P. Maroni, Le calcul des formes linéaires et les polynômes orthogonaux semi-classiques, in: Orthogonal polynomials and their applications (Segovia, 1986), 279-290, Lecture Notes in Math., 1329, Springer, Berlin, 1988. 
[62] P. Maroni, Prolégomènes à l'étude des polynômes orthogonaux semi-classiques, Ann. Mat. Pura Appl. (4) 149 (1987), 165-184.

[63] P. Maroni, Une caractérisation des polynômes orthogonaux semi-classiques, C.R. Acad. Sci. Paris Ser. I Math. 301 (1985), 269-272.

[64] P. Maroni, Les polynômes orthogonaux auto-associés modulo deux, Portugal. Math. 42 (1983/84), 195--202.

[65] P. Nevai and W. Van Assche, Compact perturbations of orthogonal polynomials, Pacific J. Math. 153 (1992), 163-184.

[66] F. Peherstorfer, Finite perturbations of orthogonal polynomials, J. Comput. Appl. Math. 44 (1992), 275-302.

[67] O. Perron, Die Lehre von den Kettenbrüchen II, B. G. Teubner, Stuttgart, (1957).

[68] E. Prianes, Polinomios ortogonales de Laguerre-Hahn, PhD Thesis, Univ. Complutense de Madrid, 1996 (in Spanish).

[69] E. Prianes and F. Marcellán, Orthogonal polynomials and Stieltjes functions: the LaguerreHahn case. Rend. Mat. Appl. 16 (1996), 117--141.

[70] J. Radon, Zum Problem von Lagrange, Hamburger mathematische Einzelschriften., 6. hft., Leipzig, 1928.

[71] M.N. Rebocho, On Laguerre-Hahn affine orthogonal polynomials on the unit circle from matrix Sylvester equations, Integral Transforms Spec. Funct. 27 (2016), 78-93.

[72] A. Ronveaux, 4th order differential equations and orthogonal polynomials of the LaguerreHahn class. Orthogonal polynomials and their applications (Erice, 1990), 379--385, IMACS Ann. Comput. Appl. Math., 9, Baltzer, Basel, 1991.

[73] A. Ronveaux, S. Belmehdi, J. Dini, and P. Maroni, Fourth-order differential equation for the co-modified of semi-classical orthogonal polynomials. J. Comput. Appl. Math. 29 (1990), $225-231$.

[74] A. Ronveaux, Fourth-order differential equations for numerator polynomials, J. Phys. A 21 (1988), L749-L753.

[75] A. Ronveaux and F. Marcellán, Co-recursive orthogonal polynomials and fourth-order differential equation, J. Comput. Appl. Math. 25 (1989), 105-109.

[76] A. Ronveaux and W. Van Assche, Upward extension of the Jacobi matrix for orthogonal polynomials, J. Approx. Theory 86 (1996), 335-357.

[77] M. Sghaier and M. Zaatra, A large family of symmetric Laguerre-Hahn polynomials of class two, Integral Transforms Spec. Funct. 22 (2011), 217--231.

[78] H.A. Slim, On co-recursive orthogonal polynomials and their application to potential scattering, J. Math. Anal. Appl. 136 (1988), 1--19.

[79] T.J. Stieltjes, Collected Papers, G. van Dijk, Ed. (Springer, Berlin, 1993).

[80] T.J. Stieltjes, Recherches sur les fractions continues, Ann. Fac. Sci. Toulouse 8 (1894) 51-122; Ann Fac. Sci. Toulouse 9 (1895) Al-47.

[81] M.H. Stone, Linear transformations in Hilbert space and their applications to Analysis, Amer Math Soc Colloq. Publ., XV, American Mathematical Society, Providence, R.I., 1932.

[82] G. Szegö, Orthogonal polynomials. 4th ed. Providence Rhode Island: Amer Math Soc Colloq. Publ. vol 23, 1975.

[83] G. Valent and W. Van Assche, The impact of Stieltjes' work on continued fractions and orthogonal polynomials: additional material, J. Comput. Appl. Math. 65 (1995), 419 - 447.

[84] W. Van Assche, Orthogonal polynomials and Painlevé equations. Australian Mathematical Society Lecture Series, 27. Cambridge University Press, Cambridge, 2018.

[85] W. Van Assche, Discrete Painlevé equations for recurrence coefficients of orthogonal polynomials. In: Elaydi S, Cushing J, Lasser R, Ruffing A, Papageorgiou V, Van Assche W, editors. 
Difference equations, special functions and orthogonal polynomials. Hackensack, NJ: World Scientific; 2007. pp. 687-725.

[86] W. Van Assche, Orthogonal polynomials, associated polynomials and functions of the second kind, J. Comput. Appl. Math. 37 (1991) 237--249.

[87] W. Van Assche, Asymptotics for Orthogonal Polynomials, Lecture Notes in Mathematics, vol. 1265, Springer-Verlag, Berlin, 1987.

[88] J. Wimp, Explicit formulas for the associated Jacobi polynomials and some applications, Canad. J. Math. 39 (1987), 983-1000.

[89] A. Wintner, Spektraltheorie der unendlichen Matrizen, Hirzel, Leipzig, 1929.

[90] A. Zhedanov, Rational spectral transformations and orthogonal polynomials, J. Comput. Appl. Math. 85 (1997), 67--86.

[91] M. Zhu and Y. Chen, On the properties of a deformed Freud weight, Random Matrices Theory Appl., 8 (2019) 1950004 (36 pp.).

M.N. REBOCHO

Departamento de Matemática, Universidade da Beira interior, 6201-001 Covilhã, Portugal; CMUC, Department of Mathematics, University of Coimbra, 3001-501 Coimbra, Portugal.

E-mail address: mneves@ubi.pt 